This item was submitted to Loughborough's Research Repository by the author.

Items in Figshare are protected by copyright, with all rights reserved, unless otherwise indicated.

\title{
A linear programming approach to efficiency evaluation in nonconvex metatechnologies
}

\section{PLEASE CITE THE PUBLISHED VERSION}

https://doi.org/10.1016/j.ejor.2018.01.013

\section{PUBLISHER}

(c) Elsevier

VERSION

AM (Accepted Manuscript)

\section{PUBLISHER STATEMENT}

This paper was accepted for publication in the journal European Journal of Operational Research and the definitive published version is available at https://doi.org/10.1016/j.ejor.2018.01.013

LICENCE

CC BY-NC-ND 4.0

\section{REPOSITORY RECORD}

Afsharian, Mohsen, and Victor Podinovski. 2018. "A Linear Programming Approach to Efficiency Evaluation in Nonconvex Metatechnologies”. figshare. https://hdl.handle.net/2134/28051. 


\title{
A linear programming approach to efficiency evaluation in nonconvex metatechnologies
}

\author{
Mohsen Afsharian \\ Corresponding author. Department of Business Sciences, Technische Universität Braunschweig, \\ Fallersleber-Tor-Wall 23, 38100 Braunschweig, Germany. Phone: +495313913606. \\ Email:m.afsharian@tu-bs.de
}

\author{
Victor V. Podinovski \\ School of Business and Economics, Loughborough University, \\ Loughborough LE11 3TU, UK. Email: v.podinovski@lboro.ac.uk
}

\begin{abstract}
The notions of metatechnology and metafrontier arise in applications of data envelopment analysis (DEA) in which decision making units (DMUs) are not sufficiently homogeneous to be considered as operating in the same technology. In this case, DMUs are partitioned into different groups, each operating in the same technology. In contrast, the metatechnology includes all DMUs and represents all production possibilities that can in principle be achieved in different production environments. Often, the metatechnology cannot be assumed to be a convex set. In such cases benchmarking a DMU against the common metafrontier requires implementing either an enumeration algorithm and solving a linear program at each of its steps, or solving an equivalent mixed integer linear program. In this paper we show that the same task can be accomplished by solving a single linear program. We also show that its dual can be used for the returns-to-scale characterization of efficient DMUs on the metafrontier.
\end{abstract}

Keywords: Data envelopment analysis; Nonconvex metatechnology; Metafrontier; Returns to scale 


\section{Introduction}

Homogeneity of decision making units (DMUs) is a common assumption made in standard applications of data envelopment analysis (DEA). This assumption allows the DMUs to be regarded as members of the same production technology, which in turn allows the DMUs to be benchmarked with respect to the common production frontier that such DMUs generate (Cooper et al., 2007). The homogeneity assumption usually means that all DMUs have similar access to the same type of resources (inputs) and produce the same range of products or services (outputs). It also implies that the operating environments of all DMUs are sufficiently similar for the purposes of efficiency evaluation (Dyson et al., 2001).

In many applications, the assumption of homogeneity may be problematic. O'Donnell et al. (2008) give several possible reasons for this, including differences in access to labour and financial capital, access to markets, natural environment, and other environmental characteristics.

For applications in which the homogeneity of DMUs cannot be accepted, Battese et al. (2004) and, specifically for DEA, O'Donnell et al. (2008) develop the metafrontier approach that enables analysis of efficiency of heterogeneous DMUs. According to this methodology, all DMUs are classified into several groups. DMUs in the same group are considered sufficiently homogeneous and represent the same group technology. The latter are usually modelled as the constant (CRS) or variable (VRS) returns-to-scale technologies (Charnes et al., 1978; Banker et al., 1984). The withingroup efficiency of DMUs is measured against the corresponding group technology frontier.

The metatechnology includes all production possibilities achievable in different environments. In particular, it includes each of the above group technologies as a subset. The boundary of the metatechnology is referred to as the metafrontier. The latter can be viewed as representing the best production possibilities that can be achieved in principle, by assuming that the operating environment for DMUs can be changed.

The efficiency of a DMU measured against the metafrontier is referred to as its metaefficiency. O'Donnell et al. (2008) suggest that the gap between the within-group efficiency and its meta-efficiency is interpretable as an indicator of the restrictive nature of the group's operating environment. Kerstens et al. (2015) provide an updated overview and discussion of the metafrontier methodology.

In recent years, the use of metafrontiers has become well-established in DEA. As highlighted by O'Donnell et al. (2008), there are two distinctly different ways in which the metatechnology, and the metafrontier, could be defined, and there are reported applications of DEA that follow both definitions.

First, the metatechnology may be defined as, for example, the single VRS or CRS technology generated by all DMUs from all groups. This approach results in a convex metatechnology. For this approach, calculation of meta-efficiency is unproblematic and requires solving a standard VRS or 
CRS model on the data set that includes all DMUs in all groups. For example, this approach was implemented by Kontolaimou and Tsekouras (2010), Portela et al. (2011), Zhang et al. (2013) and Zhang and Wei (2015).

Second, the metatechnology may be defined as the union of all group technologies. Even though each group technology may be a convex set, the metatechnology defined as the union of such sets is generally not convex. For this approach, the meta-efficiency of each DMU can be obtained by either implementing an enumeration algorithm, each step of which requires solving a linear program, or solving an equivalent mixed integer linear program (Cooper et al., 2007; Huang et al., 2013; Tiedemann et al., 2011). ${ }^{1}$

As argued by Cooper et al. (2007, page 231), Tiedemann et al. (2011) and Asmild (2015), assuming that the group technologies are convex (which is implied by the VRS or CRS models) does not mean that convex combinations of DMUs from different groups are meaningful. In particular, Asmild (2015) notes that the interpretation of benchmarks located on the metafrontier and constructed from DMUs from different operating environments may be problematic. Kerstens et al. (2015) argue that the large majority of reported applications use a convex metatechnology, which may result in a "potentially poor approximation of the metafrontier" and introduce bias in the evaluation of metaefficiency.

It may therefore appear that the approach to modelling the metatechnology which does not assume convexity between groups, should, as argued for by Kerstens et al. (2015), be more widely acceptable in DEA applications. However, this approach is computationally less straightforward than the approach for which the meta-efficiency is evaluated by solving a single conventional VRS or CRS model, using standard DEA software.

In our paper, we address the above problem of practical use of nonconvex metatechnologies, by developing a linear programming approach for the evaluation of meta-efficiency of DMUs, without assuming convexity between groups. ${ }^{2}$ From a practical perspective, this approach should be attractive because, for each DMU under the evaluation, we solve only one linear program which always has a finite optimal solution. In contrast, using a standard enumeration algorithm for the same purpose requires solving several linear programs, one for each group technology, and correctly processing all occurrences of unbounded optimal solutions or infeasibility notifications, which needs certain programming expertise and adds to the complexity of batch processing algorithms. In other words, the advantage of the proposed approach is in the simplicity of its practical application.

The suggested linear programming approach allows the dual formulation which has a meaningful interpretation. In DEA, the dual is often used for the returns-to-scale (RTS)

\footnotetext{
${ }^{1}$ De Witte and Marques (2009) use the metafrontier approach for Free Disposal Hull (FDH) group technologies (Deprins et al., 1984). In this case the metatechnology is also nonconvex.

${ }^{2}$ Our paper can be seen as continuing the tradition of linearizing different nonconvex production technologies, such as FDH. Examples of such approaches are discussed by Agrell and Tind (2001) and Leleu (2006).
} 
characterization of DMUs in the VRS technology. We show that, for metafrontiers, the RTS characterization depends on the set of group frontiers on which the DMU under the evaluation is located. We show how the dual linear program can be used for the identification of all such group frontiers, which we further use to introduce a practical approach for the RTS characterization of efficient metafrontiers.

This paper is structured as follows. In Section 2, we review the idea of modelling the metatechnology as a nonconvex set and briefly introduce the existing enumeration and mixed integer linear programming approaches to efficiency evaluation in this context. In Section 3, we develop a new linear programming approach to efficiency evaluation in a nonconvex metatechnology. In Section 4, we obtain the equivalent dual multiplier programs and discuss their meaning. In Section 5, we develop a modification of the dual program that allows us to identify all group frontiers on which the DMU is projected. In Section 6, we make further use of the dual by developing the notion of scale elasticity and returns-to-scale characterization of DMUs on the metafrontier. In Section 7, we extend our results to evaluation approaches based on directional distance functions. In Section 8, we present a numerical example illustrating the calculation of scale elasticity and assessment of returns to scale for a metafrontier. In Section 9, we summarize our contribution and outline further research avenues.

\section{Preliminaries}

Let a number of observed DMUs, be involved in a production process, characterized by inputs $i=1, \ldots, m$ and outputs $r=1, \ldots, s$. Suppose that these DMUs operate under generally different conditions that have an effect on their production performance. Depending on the context, these may include natural, labour or regulatory environments, different access to resources, and other characteristics. To represent such differences, we assume that all DMUs can be partitioned into $G>1$ distinct groups, so that DMUs in the same group $g \in \mathcal{G}=\{1, \ldots, G\}$ operate in similar conditions.

Let each group $g \in \mathcal{G}$ include DMUs $\left(X_{j}^{g}, Y_{j}^{g}\right) \in \mathfrak{R}_{+}^{m} \times \mathfrak{R}_{+}^{s}, \quad j=1, \ldots, \delta_{g}$, where $X_{j}^{g}=\left(x_{1 j}^{g}, x_{2 j}^{g}, \ldots, x_{m j}^{g}\right)$ and $Y_{j}^{g}=\left(y_{1 j}^{g}, y_{2 j}^{g}, \ldots, y_{s j}^{g}\right)$ are nonnegative and nonzero vectors ${ }^{3}$ of inputs and outputs, respectively.

Following O'Donnell et al. (2008), we view each group of DMUs as operating in a different production technology $T^{g}, g \in \mathcal{G}$. To be specific, below we assume that all such technologies are VRS technologies. This assumption is not essential: we comment on technologies for other returns-toscale assumptions in Remark 2 below. Using the conventional model of a VRS technology (Banker et al., 1984), we define each group technology as follows.

\footnotetext{
${ }^{3}$ This allows some, but not all, components of vectors $X_{j}^{g}$ and $Y_{j}^{g}$ to be equal to zero.
} 
Definition 1. Technology $T^{g}, g \in \mathcal{G}$, is the set of pairs of vectors $(X, Y) \in \mathfrak{R}_{+}^{m} \times \mathfrak{R}_{+}^{s}$ for which there exists a vector $\lambda \in \mathfrak{R}_{+}^{\delta_{g}}$ such that the following conditions are true:

$$
\begin{array}{ll}
\sum_{j=1}^{\delta_{g}} \lambda_{j}^{g} x_{i j}^{g} \leq x_{i}, & \forall i \\
\sum_{j=1}^{\delta_{g}} \lambda_{j}^{g} y_{r j}^{g} \geq y_{r}, & \forall r \\
\sum_{j=1}^{\delta_{g}} \lambda_{j}^{g}=1 . &
\end{array}
$$

Let $\mathrm{DMU}_{o}$ denote the DMU $\left(X_{o}^{q}, Y_{o}^{q}\right)$ which belongs to technology $T^{q}, q \in \mathcal{G}$, and whose efficiency is being evaluated. To be specific, below we consider the case of input radial efficiency. The case of output radial efficiency is similar and is only briefly discussed in Remark 3 below.

The within-group input radial efficiency of $\mathrm{DMU}_{o}$ may be evaluated against the frontier of any individual technology $T^{g}, g \in \mathcal{G}$. It is found as the optimal value of the following linear program:

$$
\begin{aligned}
& \operatorname{Eff}^{g}\left(X_{o}^{q}, Y_{o}^{q}\right)=\min \theta \\
& \text { s.t. } \quad \sum_{j=1}^{\delta_{g}} \lambda_{j}^{g} x_{i j}^{g} \leq x_{i o}^{q} \theta, \quad \forall i \\
& \sum_{j=1}^{\delta_{g}} \lambda_{j}^{g} y_{r j}^{g} \geq y_{r o}^{q}, \quad \forall r \\
& \sum_{j=1}^{\delta_{g}} \lambda_{j}^{g}=1, \\
& \lambda_{j}^{g} \geq 0, \forall j, \theta \text { free in sign. }
\end{aligned}
$$

Remark 1. If $\left(X_{o}^{q}, Y_{o}^{q}\right) \in T^{g}$, program (1) has a finite optimal solution and we have $\operatorname{Eff}^{g}\left(X_{o}^{q}, Y_{o}^{q}\right) \leq 1$. If $\left(X_{o}^{q}, Y_{o}^{q}\right) \notin T^{g}$, then either program (1) is infeasible or its optimal value is strictly greater than 1 .

The metatechnology for the group of technologies $T^{g}, g \in \mathcal{G}$, is defined as follows:

$$
T^{M}=T^{1} \cup T^{2} \cup \ldots \cup T^{G} .
$$

Even though each technology $T^{g}, g \in \mathcal{G}$, is convex, the metatechnology $T^{M}$ is generally not a convex set. Assessing the input radial efficiency of $\mathrm{DMU}_{o}$ against the metafrontier (frontier of technology $\left.T^{M}\right)$ is equivalent to finding the minimum of the within-group efficiencies $\operatorname{Eff}^{g}\left(X_{o}^{q}, Y_{o}^{q}\right)$, across all technologies $T^{g}$ such that $\left(X_{o}^{q}, Y_{o}^{q}\right) \in T^{g}, g \in \mathcal{G}$ :

$$
\operatorname{Eff}^{M}\left(X_{o}^{q}, Y_{o}^{q}\right)=\min \left\{\operatorname{Eff}^{g}\left(X_{o}^{q}, Y_{o}^{q}\right) \mid\left(X_{o}^{q}, Y_{o}^{q}\right) \in T^{g}, g \in \mathcal{G}\right\} .
$$

Formula (3) suggests an enumeration algorithm for calculating the meta-efficiency of DMU $\left(X_{o}^{q}, Y_{o}^{q}\right)$. For each $g \in \mathcal{G}$, we solve linear program (1) and either identify the corresponding within- 
group efficiency $\operatorname{Eff}^{g}\left(X_{o}^{q}, Y_{o}^{q}\right)$ or establish that DMU $\left(X_{o}^{q}, Y_{o}^{q}\right) \notin T^{g}$ (see Remark 1). We subsequently take the minimum of all of the obtained within-group efficiencies, across all groups $g$ such that $\left(X_{o}^{q}, Y_{o}^{q}\right) \in T^{g}$.

Cooper et al. (2007, page 231) present a single mixed integer linear program (MILP) equivalent to the above enumeration algorithm. A variant of this program is also used by Huang et al. (2013). These programs incorporate a set of binary variables, each corresponding to a particular group $g \in \mathcal{G}$. In any feasible solution, only one of such binary variables, corresponding to, say, $g=g^{*}$, is equal to 1 , while the remaining binary variables are equal to zero. For each such $g$, the MILP program becomes program (1). Effectively, this approach is a compact statement of the enumeration algorithm in the form of a single MILP program.

Example 1. Figure 1 shows three group VRS technologies $T^{1}, T^{2}$ and $T^{3}$ in two input dimensions. The single output is assumed to be the same for all DMUs, and is not shown. The boundaries of technologies $T^{1}, T^{2}$ and $T^{3}$ are, respectively, $A B C D Q, F G H J R$ and KLMNP. The metatechnology $T^{M}$ defined as in (2) is the union of the three group technologies and is shown as the shaded area above the metafrontier ABCRHSMNP.

Figure 1. The metatechnology in Example 1

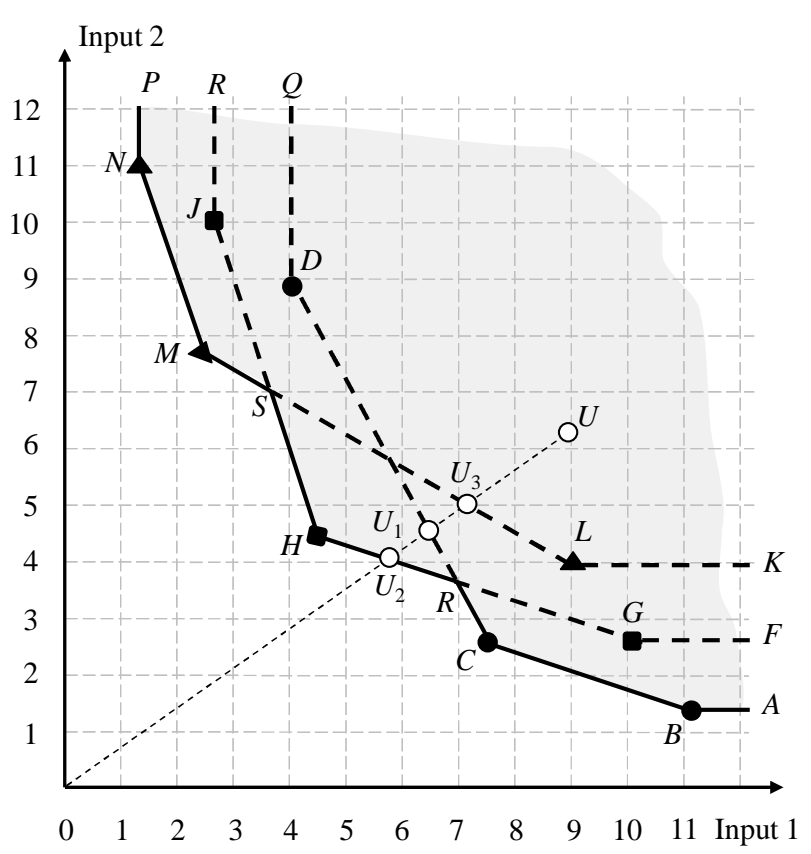

Consider assessing the input radial efficiency of DMU $U$. Because this DMU is a member of all three group technologies, its within-group efficiency (1) is defined for each of them. Namely, $E f f^{1}(U)=O U_{1} / O U, E f f^{2}(U)=O U_{2} / O U$, and $E f f^{3}(U)=O U_{3} / O U$. According to formula (3), the meta-efficiency of DMU $U$ is equal to the minimum of the above three ratios, i.e., $E f f^{M}(U)=O U_{2} / O U$, and the input radial projection of $U$ on the metafrontier is DMU $U_{2}$. 


\section{The suggested approach}

\subsection{An intermediate nonlinear program}

We start by noting that the enumeration algorithm based on formula (3) for the assessment of input radial meta-efficiency of DMU $\left(X_{o}^{q}, Y_{o}^{q}\right)$ can be equivalently stated by the following mixed integer nonlinear program:

$$
\begin{aligned}
& \operatorname{Eff}^{M}\left(X_{o}^{q}, Y_{o}^{q}\right)=\min \sum_{g=1}^{G} \theta^{g} \\
& \text { s.t. } \quad \beta^{g} \sum_{j=1}^{\delta_{g}} \lambda_{j}^{g} x_{i j}^{g} \leq x_{i o}^{q} \theta^{g}, \quad \forall g, i \\
& \beta^{g} \sum_{j=1}^{\delta_{g}} \lambda_{j}^{g} y_{r j}^{g} \geq y_{r o}^{q} \beta^{g}, \quad \forall g, r \\
& \beta^{g} \sum_{j=1}^{\delta_{g}} \lambda_{j}^{g}=\beta^{g}, \quad \forall g \\
& \sum_{g=1}^{G} \beta^{g}=1 \\
& \beta^{g} \in\{0,1\}, \lambda_{j}^{g} \geq 0, \theta^{g} \text { free in sign, } \quad \forall g, j .
\end{aligned}
$$

Because variables $\beta^{g}$ are binary, equality (4.5) implies that, in any feasible solution of program (4), exactly one variable $\beta^{g^{*}}=1$ and, therefore, $\beta^{g}=0$, for all $g \neq g^{*}$.

Consider any $g^{*} \in \mathcal{G}$ such that $\left(X_{o}^{q}, Y_{o}^{q}\right) \in T^{g^{*}}$. Because $\left(X_{o}^{q}, Y_{o}^{q}\right) \in T^{q}$ and $q \in \mathcal{G}$, we have at least one such $g^{*}$. For $g=g^{*}$, constraints $(4.2)-(4.4)$ are the constraints of program (1). The minimal value $\theta^{g^{*}}$ that satisfies these constraints is the within-group efficiency $\operatorname{Eff}^{g^{*}}\left(X_{o}^{q}, Y_{o}^{q}\right)$. Furthermore, for any $g \neq g^{*}$, constraints (4.2) - (4.4) are trivially satisfied by the zero vector $\lambda^{g}$ and $\theta^{g}=0$. Therefore, for the above $g^{*}$, the minimum value of the objective function in (4.1) is equal to $\operatorname{Eff}^{g^{*}}\left(X_{o}^{q}, Y_{o}^{q}\right) \leq 1$.

Now consider any $g^{*} \in \mathcal{G}$ such that $\left(X_{o}^{q}, Y_{o}^{q}\right) \notin T^{g^{*}}$. By Remark 1, in this case program (4) either does not have a feasible solution with $\beta^{g^{*}}=1$ or the corresponding minimum value of its objective function is equal to $\theta^{g^{*}}>1$. Therefore, the optimal value of program (4) cannot be attained at a feasible solution with $\beta^{g^{*}}=1$, such that $\left(X_{o}^{q}, Y_{o}^{q}\right) \notin T^{g^{*}}$.

The above implies that the optimum value of program (4) is equal to the minimum of the within-group efficiencies $\operatorname{Eff}^{g}\left(X_{o}^{q}, Y_{o}^{q}\right)$, calculated for all $g \in \mathcal{G}$ such that $\left(X_{o}^{q}, Y_{o}^{q}\right) \in T^{g}{ }^{4}$ This

\footnotetext{
${ }^{4}$ Because we assume that $\left(X_{o}^{q}, Y_{o}^{q}\right) \in T^{q}$ and $q \in \mathcal{G}$, at least one such $g=q$ exists.
} 
coincides with formula (3) and proves that program (4) correctly assesses the meta-efficiency of $\operatorname{DMU}\left(X_{o}^{q}, Y_{o}^{q}\right)$.

Below we show that program (4) can be transformed to a linear program. The idea of this transformation is the substitution of variables $\tilde{\lambda}_{j}^{g}=\beta^{g} \lambda_{j}^{g}$, for all $g \in \mathcal{G}$ and $j=1, \ldots, n$, which transforms nonlinear inequalities (4.2) and (4.3) to linear inequalities. We also show that we can replace the condition that variables $\beta^{g}$ are binary by the standard nonnegativity conditions.

To facilitate further discussion, we note that, if in an optimal solution $\hat{\Gamma}=\left\langle\hat{\theta}, \hat{\beta}, \hat{\lambda}^{g} \mid g \in \mathcal{G}\right\rangle$ to program (4) we have $\hat{\beta}^{g}=0$ (and therefore, $\hat{\theta}^{g}=0$ ), the vector $\hat{\lambda}^{g}$ becomes an arbitrary vector whose exact components do not affect the optimality of $\hat{\Gamma}$. It is clear that we can always redefine $\hat{\Gamma}$ by changing such vectors $\hat{\lambda}^{g}$ to zero vectors, while preserving the optimality of the resulting solution. This leads to the following definition.

Definition 2. $\hat{\Gamma}=\left\langle\hat{\theta}, \hat{\beta}, \hat{\lambda}^{g} \mid g \in \mathcal{G}\right\rangle$ is a proper optimal solution to program (4) if $\hat{\beta}^{g}=0$ implies $\hat{\lambda}^{g}=0$, for all $g \in \mathcal{G}$.

\subsection{The linear program}

Consider the following linear program:

$$
\begin{aligned}
& \widehat{E f f}^{M}\left(X_{o}^{q}, Y_{o}^{q}\right)=\min \sum_{g=1}^{G} \theta^{g} \\
& \text { s.t. } \quad \sum_{j=1}^{\delta_{g}} \lambda_{j}^{g} x_{i j}^{g} \leq x_{i o}^{q} \theta^{g}, \quad \forall g, i \\
& \sum_{j=1}^{\delta_{g}} \lambda_{j}^{g} y_{r j}^{g} \geq y_{r o}^{q} \beta^{g}, \quad \forall g, r \\
& \sum_{j=1}^{\delta_{g}} \lambda_{j}^{g}=\beta^{g}, \quad \forall g \\
& \sum_{g=1}^{G} \beta^{g}=1, \\
& \beta^{g} \geq 0, \lambda_{j}^{g} \geq 0, \theta^{g} \text { free in sign, } \quad \forall g, j .
\end{aligned}
$$

Because $\mathrm{DMU}_{o}$ is in technology $T^{q}$, the linear program (5) is feasible (with $\beta^{q}=1$ ). By inequalities (5.2), all components of vector $\theta$ are nonnegative. Therefore, the objective function (5.1) is bounded below and, consequently, program (5) has an optimal solution. 
Definition 3. $\tilde{\Gamma}=\left\langle\tilde{\theta}, \tilde{\beta}, \tilde{\lambda}^{g} \mid g \in \mathcal{G}\right\rangle$ is an elementary optimal solution to program (5) corresponding to group $g^{*} \in \mathcal{G}$, if vector $\tilde{\beta}$ has a single strictly positive component $\tilde{\beta}^{g^{*}}$. (Taking into account equality (5.5), we always have $\tilde{\beta}^{g^{*}}=1$.)

Theorem 1. Let $\tilde{\Gamma}=\left\langle\tilde{\theta}, \tilde{\beta}, \tilde{\lambda}^{g} \mid g \in \mathcal{G}\right\rangle$ be any optimal solution of program (5). Then, for each $g \in \mathcal{G}$,

(i) $\tilde{\beta}^{g}=0$ implies $\tilde{\theta}^{g}=0$ and $\tilde{\lambda}_{j}^{g}=0$, for all $j=1, \ldots, \delta_{g}$,

(ii) $\tilde{\beta}^{g}>0$ implies $\widehat{E f f}^{M}\left(X_{o}^{q}, Y_{o}^{q}\right)=\tilde{\theta}^{g} / \tilde{\beta}^{g}$.

Corollary 1. Let $\tilde{\Gamma}$ be an elementary optimal solution to (5) corresponding to group $g^{*} \in \mathcal{G}$. Then $\tilde{\theta}^{g^{*}}=\widehat{E f f}^{M}\left(X_{o}^{q}, Y_{o}^{q}\right)$, and $\tilde{\theta}^{g}=0$, for all $g \in \mathcal{G}, g \neq g^{*}$.

The following theorem establishes that, instead of assessing the meta-efficiency of $\mathrm{DMU}_{o}$ by solving the nonlinear program (4), which is equivalent to the definition of meta-efficiency by formula (3), we can equivalently solve the linear program (5).

Theorem 2. The following statements are true.

(i) The optimal value of program (5) is equal to the optimal value of program (4), i.e., $\operatorname{Eff}^{M}\left(X_{o}^{q}, Y_{o}^{q}\right)=\widehat{E f f}^{M}\left(X_{o}^{q}, Y_{o}^{q}\right)$.

(ii) Any proper optimal solution $\hat{\Gamma}=\left\langle\hat{\theta}, \hat{\beta}, \hat{\lambda}^{g} \mid g \in \mathcal{G}\right\rangle$ of program (4) is an elementary optimal solution of program (5).

(iii) Any elementary optimal solution $\tilde{\Gamma}=\left\langle\tilde{\theta}, \tilde{\beta}, \tilde{\lambda}^{g} \mid g \in \mathcal{G}\right\rangle$ of program (5) is a proper optimal solution of program (4).

(iv) Let $\tilde{\Gamma}=\left\langle\tilde{\theta}, \tilde{\beta}, \tilde{\lambda}^{g} \mid g \in \mathcal{G}\right\rangle$ be any optimal solution to program (5). Define the set $\mathcal{G}^{*}=\left\{g \mid \tilde{\beta}^{g}>0, g \in \mathcal{G}\right\}$. Then $\tilde{\Gamma}$ is the convex combination of $\left|\mathcal{G}^{*}\right|$ elementary optimal solutions $\Gamma_{g}$ to program (5), each corresponding to a different group $g \in \mathcal{G}^{*}$, and taken with the weight $\tilde{\beta}^{g}$ (component of vector $\tilde{\beta}$ ).

Corollary 2. The set $\tilde{\mathcal{S}}$ of optimal solutions to program (5) is the convex hull of the set $\hat{\mathcal{S}}$ of proper optimal solutions to program (4): $\tilde{\mathcal{S}}=\operatorname{conv} \hat{\mathcal{S}}$.

Remark 2. The above results extend to the case of CRS, and also the cases of non-increasing (NIRS) and non-decreasing (NDRS) returns to scale, with some minimal adjustments. Thus, in the CRS variant of program (5), we remove the normalizing equality (5.4). In the cases of NIRS and NDRS, we replace the equality sign in (5.4) by the inequality sign " $\leq$ " and " $\geq$ ", respectively. A similar adjustment is made to constraint (4.4) of program (4). 
After the above modifications of programs (4) and (5), both Theorems 1 and 2, and their Corollaries remain true in the case of CRS (and also, NIRS and NDRS). The proofs of these statements given in the Appendix are equally applicable in all these cases.

Remark 3. It is also straightforward to extend our results to the case of output radial efficiency. To transform model (5) to its output-oriented analogue, we swap $\theta^{g}$ and $\beta^{g}$ on the right-hand side of inequalities (5.2) and (5.3) (making them equal to $x_{i o}^{q} \beta^{g}$ and $y_{r o}^{q} \theta^{g}$, respectively), and also change the minimization of the objective function in (5.1) to its maximization. Program (4) is changed in a similar way. The output radial efficiency of $\mathrm{DMU}_{o}$ with respect to the metafrontier is equal to the inverse of the optimal value of either of the resulting programs. Both Theorems 1 and 2, and their Corollaries remain true with just minor obvious modifications.

\subsection{Alternative optimal solutions}

It is clear that, in most practical applications, the input radial projection of $\mathrm{DMU}_{o}$ on the metafrontier occurs on a single individual frontier of some technology $T^{g}$. However, the radial projection may also occur on the intersection of several individual frontiers at the same time. The latter may be possible for several reasons. For example, different groups of observed DMUs may include several common DMUs that generate the same part of the frontier in different group technologies. Thus, in an efficiency assessment of agricultural farms, there may be common farms in the groups of wheat producing farms and organic farms. Furthermore, there may be a common minimum level of some input for DMUs in different technologies, especially if such input is measured in integer units. For example, in the assessment of schools of different types there may, perhaps accidentally, be a common minimum number of teachers, treated as input, in the data sets for different school types.

Suppose that the radial projection of $\mathrm{DMU}_{o}$ on the metafrontier is simultaneously located on several group frontiers, referred to as active group frontiers (for the given $\mathrm{DMU}_{o}$ ).

Definition 4. The frontier of group technology $T^{g}, g \in \mathcal{G}$, is active if DMU $\left(X_{p}^{q}, Y_{p}^{q}\right) \in T^{g}$ and $\operatorname{Eff}^{g}\left(X_{o}^{q}, Y_{o}^{q}\right)=\operatorname{Eff}^{M}\left(X_{o}^{q}, Y_{o}^{q}\right)$.

Denote $\mathcal{G}^{0}$ the set of all $g \in \mathcal{G}$ such that the frontier of technology $T^{g}$ is active, i.e.,

$$
\mathcal{G}^{0}=\left\{g \in \mathcal{G} \mid\left(X_{o}^{q}, Y_{o}^{q}\right) \in T^{g}, \operatorname{Eff}^{g}\left(X_{o}^{q}, Y_{o}^{q}\right)=\operatorname{Eff}^{M}\left(X_{o}^{q}, Y_{o}^{q}\right)\right\} .
$$

For brevity, we refer to the set $\mathcal{G}^{0}$ as the set of all active group frontiers. Program (5) has different elementary optimal solutions each corresponding to a single active group frontier $g \in \mathcal{G}^{0}$ (identified by component $\tilde{\beta}^{g}=1$ ). The following example clarifies this observation.

Example 2. Consider two VRS technologies with two inputs and one output. For simplicity, we assume that the output of all DMUs is the same. Figure 2 depicts these technologies in the input dimensions only. 
Figure 2. Group technologies in Example 2

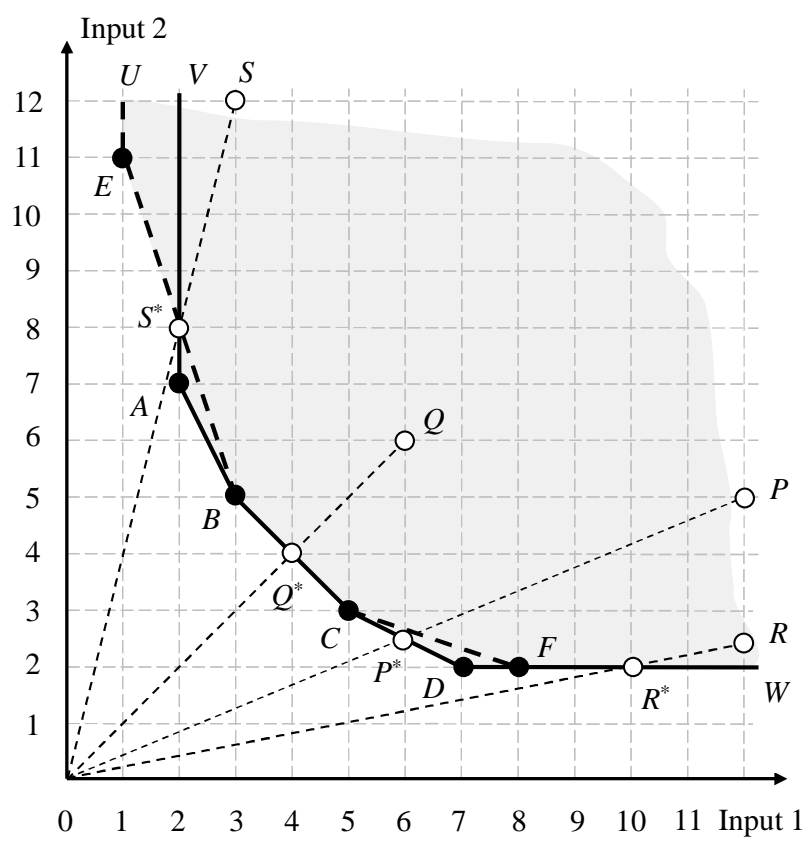

Technology $T^{1}$ is generated by observed DMUs $A, B, C$, and $D$, and its boundary is $V A B C D W$. Technology $T^{2}$ is generated by observed DMUs $E, B, C$ and $F$. Its boundary is $U E B C F W$. Note that the observed DMUs $B$ and $C$ are members of both technologies, which illustrates the first of the above scenarios. DMUs $D$ and $F$, although belonging to different technologies, have the same minimum level of Input 2, and illustrate the second scenario.

Consider assessing the input radial efficiency of DMUs $P, Q, R$ and $S$ measured against the metafrontier $U E S^{*} A B C D W$. Table 1 shows the input radial efficiency of these DMUs calculated by solving program (5) and possible alternative optimal solutions, identified by a different number in parentheses in the first column.

\begin{tabular}{|c|c|c|c|c|c|c|c|c|c|c|c|c|c|}
\hline $\begin{array}{c}\text { DMU and } \\
\text { optimal } \\
\text { solution }\end{array}$ & $\lambda_{A}^{1}$ & $\lambda_{B}^{1}$ & $\lambda_{C}^{1}$ & $\lambda_{D}^{1}$ & $\lambda_{E}^{2}$ & $\lambda_{B}^{2}$ & $\lambda_{C}^{2}$ & $\lambda_{F}^{2}$ & $\beta^{1}$ & $\beta^{2}$ & $\theta^{1}$ & $\theta^{2}$ & $\widehat{E f f}^{M}()$. \\
\hline$P$ & 0 & 0 & 0.5 & 0.5 & 0 & 0 & 0 & 0 & 1 & 0 & 0.5 & 0 & 0.5 \\
\hline$Q(1)$ & 0 & 0.5 & 0.5 & 0 & 0 & 0 & 0 & 0 & 1 & 0 & 0.6667 & 0 & 0.6667 \\
\hline$Q(2)$ & 0 & 0 & 0 & 0 & 0 & 0.5 & 0.5 & 0 & 0 & 1 & 0 & 0.6667 & 0.6667 \\
\hline$Q(3)$ & 0 & 0.1 & 0.1 & 0 & 0 & 0.4 & 0.4 & 0 & 0.2 & 0.8 & 0.1333 & 0.5333 & 0.6667 \\
\hline$R(1)$ & 0 & 0 & 0 & 0 & 0 & 0 & 0 & 1 & 0 & 1 & 0 & 0.8333 & 0.8333 \\
\hline$R(2)$ & 0 & 0 & 0 & 1 & 0 & 0 & 0 & 0 & 1 & 0 & 0.8333 & 0 & 0.8333 \\
\hline$S(1)$ & 1 & 0 & 0 & 0 & 0 & 0 & 0 & 0 & 1 & 0 & 0.6667 & 0 & 0.6667 \\
\hline$S(2)$ & 0 & 0 & 0 & 0 & 0.5 & 0.5 & 0 & 0 & 0 & 1 & 0 & 0.6667 & 0.6667 \\
\hline
\end{tabular}

Table 1. Alternative optimal solutions to program (5) for DMUs $P, Q, R$ and $S$ in the example. The first column shows the DMU under the assessment. The number in parentheses identifies a different alternative optimal solution for the corresponding DMU. 
The input radial projection of DMU $P$ on the metafrontier is DMU $P^{*}$, located on the boundary of the single technology $T^{1}$. The input radial efficiency of DMU $P$ is equal to 0.5 . In this case program (5) has a unique optimal solution.

The radial projection of DMU $Q$ is DMU $Q^{*}$ located on the boundaries of two technologies $T^{1}$ and $T^{2}$, both of which are viewed as active frontiers. As noted, in this case, program (5) has two corresponding elementary optimal solutions. The first has $\beta^{1}=1$, and the second has $\beta^{2}=1$, indicating that the projections are on the boundaries of technologies $T^{1}$ and $T^{2}$, respectively. The third solution for DMU $Q$ is the convex combination of the first two optimal solutions for $Q$, taken with the weights 0.2 and 0.8 , respectively. Note that the ratios $\theta^{1} / \beta^{1}$ and $\theta^{2} / \beta^{2}$ are both equal to the input radial efficiency 0.6667 of DMU $Q$, as stated by Theorem $1 .{ }^{5}$

The cases of DMUs $R$ and $S$ are similar, as both are projected simultaneously on the boundaries of two technologies.

The above analysis also extends to the input radial efficient DMUs. For example, DMU $B$ is located on the boundaries of both technologies $T^{1}$ and $T^{2}$. The optimal value of the corresponding program (5) is equal to 1 , and there are two elementary optimal solutions (not shown in Table 1), one with $\beta^{1}=1$ and another with $\beta^{2}=1$.

\section{The dual program}

Noting that, by Theorem 2, $\widehat{E f f}^{M}\left(X_{o}^{q}, Y_{o}^{q}\right)=E f f{ }^{M}\left(X_{o}^{q}, Y_{o}^{q}\right)$, we state the dual to program (5) as follows:

$$
\begin{aligned}
& \operatorname{Eff}^{M}\left(X_{o}^{q}, Y_{o}^{q}\right)=\max \varphi \\
& \text { s.t. } \quad \sum_{r=1}^{s} u_{r}^{g} y_{r o}^{q}+\omega^{g} \geq \varphi, \quad \forall g \\
& \sum_{i=1}^{m} v_{i}^{g} x_{i o}^{q}=1, \quad \forall g \\
& \sum_{r=1}^{s} u_{r}^{g} y_{r j}^{g}-\sum_{i=1}^{m} v_{i}^{g} x_{i j}^{g}+\omega^{g} \leq 0, \quad \forall g, j \\
& u_{r}^{g}, v_{i}^{g} \geq 0, \omega^{g}, \varphi \text { free in sign, } \quad \forall g, i, r .
\end{aligned}
$$

In this model, $v_{i}^{g}$ and $u_{r}^{g}$ are the input and output weights specific to group $g \in \mathcal{G}$. These variables are dual to the corresponding inequalities (5.2) and (5.3). Variables $\omega^{g}$ are dual to constraints (5.4), and $\varphi$ is dual to (5.5).

\footnotetext{
${ }^{5}$ It is straightforward to verify that this solution, identified as $Q(3)$ in Table 1 is indeed optimal, as stated by Corollary 2 of Theorem 2. Note that this solution is not basic and therefore cannot be obtained by solving program (5) by the simplex method.
} 
For any fixed $g \in \mathcal{G}$, maximizing $\varphi$ in program (6) is equivalent to maximizing the left-hand side of inequality (6.2). In this case program (6) becomes the standard input-oriented VRS multiplier model stated as follows:

$$
\begin{array}{ll}
\text { Eff }^{g}\left(X_{o}^{q}, Y_{o}^{q}\right)=\max & \sum_{r=1}^{s} u_{r}^{g} y_{r o}^{q}+\omega^{g} \\
\text { s.t. } & \sum_{i=1}^{m} v_{i}^{g} x_{i o}^{q}=1, \\
& \sum_{r=1}^{s} u_{r}^{g} y_{r j}^{g}-\sum_{i=1}^{m} v_{i}^{g} x_{i j}^{g}+\omega^{g} \leq 0, \quad \forall j \\
& u_{r}^{g}, v_{i}^{g} \geq 0, \forall i, r, \omega^{g} \text { free in sign. }
\end{array}
$$

Program (7) is the dual to the envelopment program (1). If DMU $\left(X_{o}^{q}, Y_{o}^{q}\right) \in T^{g}$, this program evaluates its input radial efficiency $\operatorname{Eff}^{g}\left(X_{o}^{q}, Y_{o}^{q}\right)$ in technology $T^{g}$. If $\left(X_{o}^{q}, Y_{o}^{q}\right) \notin T^{g}$, then either the optimal value of program (7) is strictly greater than 1 or its objective function is unbounded. ${ }^{6}$

Taking into account inequality (6.2), it is clear that the maximum of the objective function $\varphi$ of program (6) is equal to the minimum of all within-group efficiencies $E f f^{g}\left(X_{o}^{q}, Y_{o}^{q}\right) \leq 1$ taken over all $g \in \mathcal{G}$ such that $\left(X_{o}^{q}, Y_{o}^{q}\right) \in T^{g}$. Therefore, solving program (6) is theoretically equivalent to implementing the enumeration algorithm based on formula (3), but performed in a single step and in the dual space of the input and output weights.

Remark 4. As noted in Remark 2, in the case of CRS, we remove equalities (5.4) from program (5). In the dual programs (6) and (7), we remove the corresponding variables $\omega^{g}, g \in \mathcal{G}$. In the case of NIRS and NDRS technologies, the variables $\omega^{g}$ are subject to additional constraints $\omega^{g} \leq 0$ and $\omega^{g} \geq 0, g \in \mathcal{G}$, respectively. It is also straightforward to state an analogue of the dual program (6) in the case of output radial efficiency.

\section{Using the dual to identify active group frontiers}

In Section 3.3, we defined the set $\mathcal{G}^{0} \subseteq \mathcal{G}$ of active group frontiers for any $\mathrm{DMU}_{o}$ in the metatechnology $T^{M}$. Identifying the set $\mathcal{G}^{0}$ is important for practical applications, for example, for returns-to-scale characterization of efficient DMUs, which is considered in detail in the next section.

It might appear that the set $\mathcal{G}^{0}$ is identified by those groups $g \in \mathcal{G}$ for which the inequality (6.2) is satisfied as equality, for an arbitrary optimal solution of program (6). Let us show that this is not so, and suggest a simple modification of the dual that identifies the set $\mathcal{G}^{0}$ correctly.

\footnotetext{
${ }^{6}$ This follows from Remark 1 and the fact that program (7) is feasible for any $g \in \mathcal{G}$.
} 
Let $\Delta=\left\langle\tilde{u}^{g}, \tilde{v}^{g}, \tilde{\omega}^{g}, \tilde{\varphi} \mid g \in \mathcal{G}\right\rangle$ be any optimal solution to program (6). Denote $\mathcal{G}(\Delta)$ the corresponding set of all group technologies $g \in \mathcal{G}$ for which the inequality (6.2) is satisfied as equality.

Theorem 3. For any optimal solution $\Delta$ of program (6), we have $\mathcal{G}^{0} \subseteq \mathcal{G}(\Delta)$. Furthermore, for any set $\mathcal{G}^{*}$ such that $\mathcal{G}^{0} \subseteq \mathcal{G}^{*} \subseteq \mathcal{G}$, there exists an optimal solution $\Delta^{*}$ of program (6), for which $\mathcal{G}\left(\Delta^{*}\right)=\mathcal{G}^{*}$.

The above result implies that the set $\mathcal{G}(\Delta)$ identified by an arbitrary optimal solution $\Delta$ of program (6) always includes the set of active group frontiers $\mathcal{G}^{0}$ as a subset. However, it may also include any additional number of frontiers $g \in \mathcal{G} \backslash \mathcal{G}^{0}$. Therefore, a zero slack in inequality (6.2) for a particular $g$ does not imply that $g \in \mathcal{G}^{0}$.

A simple modification of the dual (6) allows us to overcome the above problem. Note that the optimal value of program (6) is equal to the input radial efficiency $E f f^{M}\left(X_{o}^{q}, Y_{o}^{q}\right) \leq 1$ of $\mathrm{DMU}_{o}=$ $\left(X_{o}^{q}, Y_{o}^{q}\right)$ in the metatechnology $T^{M}$. By the definition of active frontiers, for any $g \in \mathcal{G}^{0}$, the optimal value of program (7) is equal to $\operatorname{Eff}^{M}\left(X_{o}^{q}, Y_{o}^{q}\right)$. For any $g \in \mathcal{G} \backslash \mathcal{G}^{0}$, the optimal value of the program (7) is either unbounded above or is strictly greater than $\operatorname{Eff}^{M}\left(X_{o}^{q}, Y_{o}^{q}\right)$. Consider the following modification of the dual (6) that utilizes technology-specific variables $\varphi^{g}, g \in \mathcal{G}$ :

$$
\begin{aligned}
& \max \sum_{g=1}^{G} \varphi^{g} \\
& \text { s.t. } \quad \sum_{r=1}^{s} u_{r}^{g} y_{r o}^{q}+\omega^{g}=\varphi^{g}, \quad \forall g \\
& \varphi^{g} \leq L, \quad \forall g \\
& \sum_{i=1}^{m} v_{i}^{g} x_{i o}^{q}=1, \quad \forall g \\
& \sum_{r=1}^{s} u_{r}^{g} y_{r j}^{g}-\sum_{i=1}^{m} v_{i}^{g} x_{i j}^{g}+\omega^{g} \leq 0, \quad \forall g, j \\
& u_{r}^{g}, v_{i}^{g} \geq 0, \omega^{g}, \varphi^{g} \text { free in sign, } \quad \forall g, i, r .
\end{aligned}
$$

In the above program, $L$ is any constant such that $L>1$. This guarantees that $L>\operatorname{Eff}^{M}\left(X_{o}^{q}, Y_{o}^{q}\right)$. Solving program (8) is equivalent to solving programs (7), separately for each $g \in \mathcal{G}$, in which the objective function is bounded above by constant $L{ }^{7}$

\footnotetext{
${ }^{7}$ It is straightforward to show that all programs (7) with their objective functions bounded above by $L$ are feasible and hence have a finite optimal solution. Their feasibility follows from the following observation. Consider any feasible solution $\left\langle\tilde{u}^{g}, \tilde{v}^{g}, \tilde{\omega}^{g}\right\rangle$ such that the objective function of (7) is greater than $L$. Replace the vector $\tilde{u}^{g}$ and scalar $\tilde{\omega}^{g}$ by zeros. Then $\left\langle 0, \tilde{v}^{g}, 0\right\rangle$ is feasible in program (7) with the upper bound $L$ on its objective function.
} 
Let $\tilde{\Delta}=\left\langle\tilde{u}^{g}, \tilde{v}^{g}, \tilde{\omega}^{g}, \tilde{\varphi}^{g} \mid g \in \mathcal{G}\right\rangle$ be any optimal solution of program (8). Define

$$
\tilde{\varphi}=\min \left\{\tilde{\varphi}^{g} \mid g \in \mathcal{G}\right\} \text {. }
$$

Theorem 4. Solution $\tilde{\tilde{\Delta}}=\left\langle\tilde{u}^{g}, \tilde{v}^{g}, \tilde{\omega}^{g}, \tilde{\varphi} \mid g \in \mathcal{G}\right\rangle$ is optimal in program (6).

The above theorem implies that the input radial efficiency $E f f^{M}\left(X_{o}^{q}, Y_{o}^{q}\right)$ evaluated by solving the dual (6) can also be identified by solving the modified dual (8), i.e., we have

$$
\operatorname{Eff}^{M}\left(X_{o}^{q}, Y_{o}^{q}\right)=\min \left\{\tilde{\varphi}^{g} \mid g \in \mathcal{G}\right\} .
$$

Theorem 5. We have $\tilde{\varphi}^{g}=E f f{ }^{M}\left(X_{o}^{q}, Y_{o}^{q}\right)$ for $g \in \mathcal{G}^{0}$, and $\tilde{\varphi}^{g}>E f f{ }^{M}\left(X_{o}^{q}, Y_{o}^{q}\right)$ for $g \in \mathcal{G} \backslash \mathcal{G}^{0}$.

This result implies that the set of active group frontiers $\mathcal{G}^{0}$ is identified by any optimal solution $\tilde{\Delta}$ of the modified dual program (8) as the set of all $g \in \mathcal{G}$ for which $\tilde{\varphi}^{g}=\min \left\{\tilde{\varphi}^{g} \mid g \in \mathcal{G}\right\}$ where, by (10), the right-hand side is also equal to $\operatorname{Eff}^{M}\left(X_{o}^{q}, Y_{o}^{q}\right)$.

\section{Returns to scale for metafrontiers}

Similar to the case of conventional VRS technology, we can use the set of optimal solutions to the dual program (6) for the characterization of returns to scale (RTS) on the efficient frontier of metatechnology $T^{M}$.

In the VRS technology, the type of RTS exhibited by an efficient $\mathrm{DMU}_{o}$ is defined by the lefthand and right-hand scale elasticities $\varepsilon_{o}^{-}$and $\varepsilon_{o}^{+}$evaluated at this DMU. Following Banker and Thrall (1992) and Førsund and Hjalmarsson (2004), these one-sided scale elasticities can be expressed via the minimum and maximum optimal values of the sign-free variable $\omega$ in the standard input-oriented VRS multiplier model, denoted $\omega^{\min }$ and $\omega^{\max }$, respectively. ${ }^{8}$ The latter model is program (7) in which the superscript $g$ is omitted. Then

$$
\varepsilon_{o}^{-}=\frac{1}{1-\omega^{\max }}, \quad \varepsilon_{o}^{+}=\frac{1}{1-\omega^{\min }} \cdot
$$

It is known that, in the VRS technology, we have

$$
\varepsilon_{o}^{-} \geq \varepsilon_{o}^{+}{ }^{10}
$$

\footnotetext{
${ }^{8}$ The use of the input-oriented multiplier model for the calculation of scale elasticity requires that $\mathrm{DMU}_{o}$ be both input and output radial efficient. Alternative computations of scale elasticity based on the output-oriented multiplier model require that $\mathrm{DMU}_{o}$ be only output radial efficient. See Podinovski et al. (2009) and Podinovski and Førsund (2010) for details.

${ }^{9}$ In order to avoid considering special cases, we formally let $\varepsilon_{o}^{-}=+\infty$ if $\omega^{\max }=1$ and $\varepsilon_{o}^{+}=0$ if $\omega^{\min }=-\infty$. The former case implies that a marginal proportional reduction of the input vector $X_{o}^{q}$ of $\mathrm{DMU}_{o}$ is impossible as it leads outside the VRS technology (Podinovski and Førsund 2010). This situation arises when DMU ${ }_{o}$ is located on a "vertical" weakly efficient facet generated by the assumption of free disposability of outputs.

${ }^{10}$ For an arbitrary convex technology, the inequality (12) is proved by Podinovski (2017).
} 
Using the definition of RTS given by Banker and Thrall (1992), DMU $\mathrm{DM}_{o}$ exhibits IRS if $1<\varepsilon_{o}^{+} \leq \varepsilon_{o}^{-}$, DRS if $\varepsilon_{o}^{+} \leq \varepsilon_{o}^{-}<1$, and CRS if $\varepsilon_{o}^{+} \leq 1 \leq \varepsilon_{o}^{-}$. These three cases correspond to the inequalities $\omega^{\min }>0, \omega^{\max }<0$ and $\omega^{\min } \leq 0 \leq \omega^{\max }$, respectively.

Extending the notion of RTS to the metafrontier of several VRS technologies is relatively straightforward. The only difference that requires careful treatment arises in possible cases when $\mathrm{DMU}_{o}$ is located at the intersection of several (more than one) group frontiers. This possibility is illustrated by the example considered at the end of this section.

Let $\mathrm{DMU}_{o}=\left(X_{o}^{q}, Y_{o}^{q}\right)$ be efficient in metatechnology $T^{M} \cdot{ }^{11}$ Intuitively, the left-hand and right-hand scale elasticities $\varepsilon_{o}^{M-}$ and $\varepsilon_{o}^{M+}$ evaluated at $\mathrm{DMU}_{o}$ on the metafrontier should depend on the corresponding one-sided scale elasticities $\varepsilon_{o}^{g-}$ and $\varepsilon_{o}^{g+}$ evaluated on all active group frontiers $g \in \mathcal{G}^{0}$. The following theorem establishes this relationship formally.

Theorem 6. The one-sided scale elasticities $\varepsilon_{o}^{M-}$ and $\varepsilon_{o}^{M+}$ exist (we allow $\varepsilon_{o}^{M-}=+\infty$ ) and

$$
\varepsilon_{o}^{M-}=\min \left\{\varepsilon_{o}^{g-} \mid g \in \mathcal{G}^{0}\right\}, \quad \varepsilon_{o}^{M+}=\max \left\{\varepsilon_{o}^{g+} \mid g \in \mathcal{G}^{0}\right\} .
$$

Corollary 3. If $\left|\mathcal{G}^{0}\right|=1$, i.e., $\mathrm{DMU}_{o}$ belongs to a single group technology $T^{q}$, then $\varepsilon_{o}^{M-}=\varepsilon_{o}^{q-}$ and $\varepsilon_{o}^{M+}=\varepsilon_{o}^{q+}$.

According to Theorem 6, in order to calculate the one-sided scale elasticities $\varepsilon^{M-}$ and $\varepsilon^{M+}$ at $\mathrm{DMU}_{o}$, it suffices to evaluate the one-sided scale elasticities $\varepsilon^{g-}$ and $\varepsilon^{g+}$, separately for each active group frontier $g \in \mathcal{G}^{0}$, and then take their minimum and maximum, respectively. The set of all active group frontiers $\mathcal{G}^{0}$ can be found by solving program (8). For each $g \in \mathcal{G}^{0}, T^{g}$ is the standard VRS technology, and the one-sided scale elasticities $\varepsilon^{g-}$ and $\varepsilon^{g+}$ can be found by identifying the maximum and minimum values of variable $\omega^{g}$ among all optimal solutions to program (7) and applying formula (11).

Example 3. Figure 3 shows three VRS technologies, $T^{1}, T^{2}$ and $T^{3}$, with a single input and a single output. The efficient frontier of technology $T^{1}$ is the broken line $A B C D E$. The efficient frontier of technology $T^{2}$ is the line $G B H$, and the efficient frontier of technology $T^{3}$ is the line $K D L$. The efficient metafrontier is the line $G B C D L$.

\footnotetext{
${ }^{11}$ It suffices that $\mathrm{DMU}_{o}$ be both input and output radial efficient in the metatechnology $T^{M}$ (see Footnote 8).
} 
Figure 3. Group technologies in Example 3

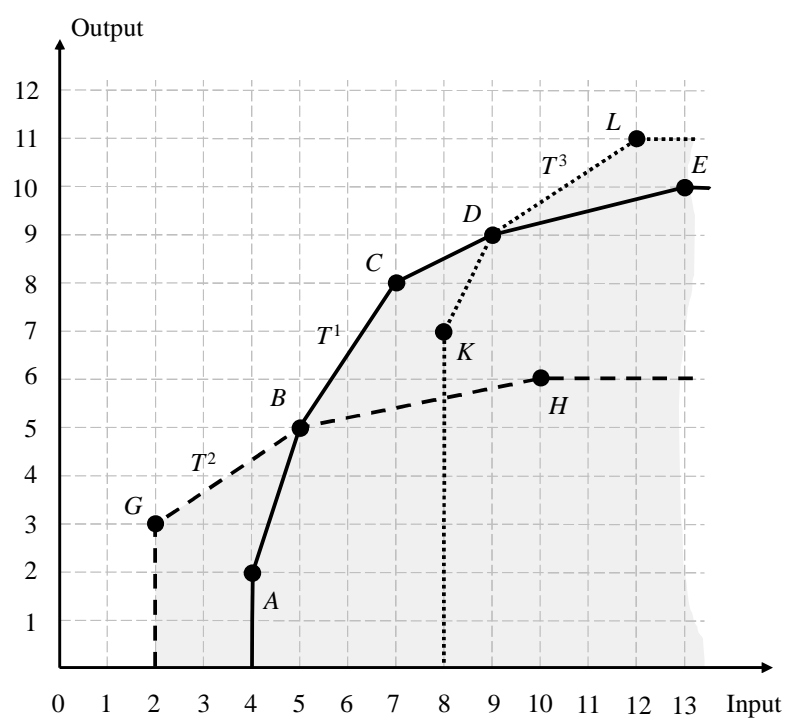

Table 2 shows the one-sided scale elasticities $\varepsilon^{M-}$ and $\varepsilon^{M+}$ evaluated at the five DMUs located on the metafrontier. DMUs $C, G$ and $L$ are located on single group frontiers. By Corollary 3 , for these DMUs, the one-sided scale elasticities $\varepsilon^{M-}$ and $\varepsilon^{M+}$ are the same as the one-sided scale elasticities $\varepsilon^{g-}$ and $\varepsilon^{g+}$ evaluated on the corresponding single group frontiers to which these DMUs belong. For example, for DMU $C$, we have $\varepsilon_{C}^{M-}=\varepsilon_{C}^{1-}=1.3125$ and $\varepsilon_{C}^{M+}=\varepsilon_{C}^{1+}=0.4375 .{ }^{12}$ Therefore, DMU $C$ exhibits CRS on the metafrontier.

\begin{tabular}{|c|c|c|c|c|}
\hline DMU & Input & Output & $\varepsilon_{0}^{M-}$ & $\varepsilon_{0}^{M+}$ \\
\hline$A$ & 4 & 2 & N/A & N/A \\
\hline$B$ & 5 & 5 & 0.6667 & 1.5 \\
\hline$C$ & 7 & 8 & 1.3125 & 0.4375 \\
\hline$D$ & 9 & 9 & 0.5 & 0.6667 \\
\hline$E$ & 13 & 10 & N/A & N/A \\
\hline$G$ & 2 & 3 & $+\infty$ & 0.4444 \\
\hline$H$ & 10 & 6 & N/A & N/A \\
\hline$K$ & 8 & 7 & N/A & N/A \\
\hline$L$ & 12 & 11 & 0.7273 & 0 \\
\hline
\end{tabular}

Table 2. DMUs in Example 3 and the one-sided scale elasticities $\varepsilon_{0}^{M-}$ and $\varepsilon_{0}^{M+}$ evaluated on the metafrontier. The notion of scale elasticity does not apply (N/A) to all DMUs that are inefficient in the metatechnology $T^{M}$.

\footnotetext{
${ }^{12}$ As an alternative to evaluating the one-sided scale elasticities by using formula (11), which requires solving two linear programs, we use the known equivalent definition of scale elasticity as the ratio of the marginal to average productivity (valid in the case of a single input and single output). For example, the right-hand marginal productivity at DMU $C$ is the slope of the line $C D$, equal to $1 / 2$. The average productivity at $C$ is the ratio of its output to input, equal to $8 / 7$. Then $\varepsilon_{C}^{1+}=(1 / 2) /(8 / 7) \approx 0.4375$. Similarly, $\varepsilon_{C}^{1-}=(3 / 2) /(8 / 7) \approx 1.3125$, where $3 / 2$ is the slope of the line $B C$.
} 
In contrast, each DMU $B$ and $D$ is located on two group frontiers. As seen from Figure 3 and is formally established by Theorem 6 , the right-hand scale elasticity $\varepsilon_{B}^{M+}$ evaluated at DMU $B$ on the metafrontier is equal to the maximum of the right-hand scale elasticities $\varepsilon_{B}^{g+}$, evaluated at this DMU on the group frontiers $g=1,2$, i.e., $\varepsilon_{B}^{M+}=\max \{1.5,0.2\}=1.5$. Similarly, the left-hand scale elasticity $\varepsilon_{B}^{M-}$ is equal to the minimum of the left-hand scale elasticities $\varepsilon_{B}^{g-}$ evaluated for group frontiers $g=1,2 \quad$, i.e., $\quad \varepsilon_{B}^{M-}=\min \{3,0.6667\}=0.6667 \quad$. For $\quad$ DMU $D$, we have $\varepsilon_{D}^{M+}=\max \{0.25,0.6667\}=0.6667$ and $\varepsilon_{D}^{M-}=\min \{0.5,2\}=0.5$.

The interpretation of one-sided scale elasticities $\varepsilon_{o}^{M-}$ and $\varepsilon_{o}^{M+}$ evaluated on the metafrontier is similar to the case of a single VRS technology, but also takes into account the meaning of the metatechnology. For example, if the input vector $X_{o}^{q}$ of $\mathrm{DMU}_{o}$ is increased in a marginal proportion, e.g., by $1 \%$, the maximum proportional increase of the output vector $Y_{o}^{q}$ in the metatechnology $T^{M}$ (i.e., assuming that $\mathrm{DMU}_{o}$ is able to operate within any group technology $T^{g}$ ), is $\varepsilon_{o}^{M+}$ percent. Similarly, if the input vector $X_{o}^{q}$ is reduced by $1 \%$, the maximum level of the output vector $Y_{o}^{q}$ available in the metatechnology is proportionally reduced by $\varepsilon_{o}^{M-}$ percent.

Using DMU $B$ as an example, increasing its input level of 5 by $1 \%$ leads to a maximum increase of its output level of 5 by $\varepsilon_{B}^{M+}=1.5 \%$. This corresponds to the modified DMU $B$ electing to stay within technology $T^{1}$ and moving along its efficient segment $B C$. However, reducing the input level of DMU $B$ by $1 \%$ would lead to the reduction of its maximum possible output by $\varepsilon_{B}^{M-}=0.6667 \%$, which corresponds to staying in technology $T^{2}$ and moving along its efficient segment $G B$.

The difference between the right-hand scale elasticity evaluated on the metafrontier and any of the active group frontiers $g^{\prime} \in \mathcal{G}^{0}$ is interpretable as the gap between the optimal output growth rate achievable on the metafrontier and the frontier of the single technology $g^{\prime}$. For example, the optimal marginal growth rate for DMU $B$ is described by $\varepsilon_{B}^{M+}=1.5 \%$ achievable if DMU $B$ follows the frontier of technology $T^{1}$. Note that the fastest marginal growth rate for DMU $B$ in technology $T^{2}$ is $\varepsilon_{B}^{1+}=0.2 \%$. The difference $1.5-0.2=1.3 \%$ quantifies the gap between the growth opportunities for DMU $B$ in the two technologies.

Similarly, the difference between the left-hand scale elasticity evaluated on any active frontier $g^{\prime} \in \mathcal{G}^{0}$ and the metafrontier identifies the gap between the output reduction rate for the frontier of the single technology $g^{\prime}$ and the metafrontier, in the case of marginal proportional input reduction. For example, if DMU $B$ marginally reduces its input, then the output is reduced by $\varepsilon_{B}^{1-}=3 \%$ on the frontier of technology $T^{1}$ and only $\varepsilon_{B}^{M-}=0.6667 \%$ on the metafrontier (which is the frontier of 
technology $T^{2}$ ). The difference $3-0.6667=2.3333 \%$ is the additional loss of output for DMU $B$ arising from staying in technology $T^{1}$ in contrast with following technology $T^{2}$, which provides the best opportunity in the metatechnology if the input is to be marginally reduced.

Let us now refer to the RTS characterization of DMUs on the metafrontier. Corollary 3 shows that the only difference between the RTS characterization on the standard VRS frontier and the metafrontier arises in the case when $\left|\mathcal{G}^{0}\right|>1$. As illustrated by DMUs $B$ and $D$, the inequality (12) may not hold for the one-sided scale elasticities $\varepsilon_{o}^{M-}$ and $\varepsilon_{o}^{M+}$ evaluated on the metafrontier. This creates various situations which are not properly categorized by the standard definition of RTS. However, in all such situations we still have correctly defined one-sided types of RTS that have a clear practical meaning.

For example, for DMU $B$, we have $\varepsilon_{B}^{M-}<1$ and $\varepsilon_{B}^{M+}>1$. Therefore, DMU $B$ exhibits DRS on the left and IRS on the right (when its input is, respectively, reduced and increased in a marginal proportion). This situation is impossible in a single group VRS technology.

Another nonstandard situation is illustrated by DMU $D$ for which we have $\varepsilon_{D}^{M-}<1$ and $\varepsilon_{D}^{M+}<1$. This means that DMU $D$ exhibits DRS for any marginal change (increase or decrease) of its input. However, because $\varepsilon_{D}^{M-}<\varepsilon_{D}^{M+}$, the DRS characterization is stronger (identified by the higher scale elasticity) on the right of DMU $D$ than on the left. This situation cannot arise in the standard VRS technology for which we always have inequality (12). A similar example can be given for the case of IRS.

Remark 5. As in the conventional case of standard VRS technology, if DMU $\left(X_{p}^{q}, Y_{p}^{q}\right) \in T^{q}$ is output radial inefficient in the metatechnology $T^{M}$, its RTS type is undefined. However, we may consider projecting this DMU on the metafrontier and apply the above classification to the projected DMU. As in the conventional single VRS technology, the resulting RTS characterization depends on the particular projection that we use, e.g., the RTS type is generally different for the input and output radial projections.

\section{Extensions for directional distance functions}

In a more general framework of measuring performance, the efficiency of a DMU can be characterized by evaluating its distance to the boundary of a benchmark technology (e.g., individual group technology or the metatechnology) along a predetermined direction which may simultaneously seek to expand the outputs and contract the inputs (Afsharian and Ahn, 2014). Below we briefly outline how the above linearization approach developed for the radial measures of efficiency can be applied to the case of directional distance functions. 
Let $T \subset \mathfrak{R}_{+}^{m} \times \mathfrak{R}_{+}^{s}$ be a production technology. Consider any DMU $(X, Y) \in \mathfrak{R}_{+}^{m} \times \mathfrak{R}_{+}^{s}$ and a user-specified direction $\left(d^{x}, d^{y}\right) \in \mathfrak{R}_{+}^{m} \times \mathfrak{R}_{+}^{s}$ such that $\left(d^{x}, d^{y}\right) \neq 0$. Following Chambers et al. (1998), the directional distance function can be defined as follows:

$$
D\left(X, Y ; d^{x}, d^{y}\right)=\sup \left\{\alpha \mid(X, Y)+\alpha\left(-d^{x}, d^{y}\right) \in T, \alpha \in \mathfrak{R}\right\} .
$$

Remark 6. If DMU $(X, Y) \in T$, then $D\left(X, Y ; d^{x}, d^{y}\right) \geq 0$, which is interpreted as a measure of DMU's inefficiency, for the chosen directions $\left(d^{x}, d^{y}\right)$. If DMU $(X, Y) \notin T$, then either $D\left(X, Y ; d^{x}, d^{y}\right)<0$ or the supremum in (13) is undefined (often formally taken to be $-\infty$ ).

Using our previous notation, suppose we wish to assess the efficiency of $\mathrm{DMU}_{o}=\left(X_{o}^{q}, Y_{o}^{q}\right)$ in the group technology $T^{g}, g \in \mathcal{G}$, using the directional distance function in (13). This leads to the following linear program:

$$
\begin{aligned}
& D^{g}\left(X_{o}^{q}, Y_{o}^{q} ; d^{x}, d^{y}\right)=\max \alpha \\
& \text { s.t. } \quad \sum_{j=1}^{\delta_{g}} \lambda_{j}^{g} x_{i j}^{g} \leq x_{i o}^{q}-\alpha d_{i}^{x}, \quad \forall i \\
& \sum_{j=1}^{\delta_{g}} \lambda_{j}^{g} y_{r j}^{g} \geq y_{r o}^{q}+\alpha d_{r}^{y}, \quad \forall r \\
& \sum_{j=1}^{\delta_{g}} \lambda_{j}^{g}=1, \\
& \lambda_{j}^{g} \geq 0, \forall j, \alpha \text { free in sign. }
\end{aligned}
$$

Assessing the efficiency of $\mathrm{DMU}_{o}$ against the metafrontier (i.e., in the metatechnology $T^{M}$ ) using the directional distance function (13) leads to the following mixed integer nonlinear program similar to program (4):

$$
\begin{aligned}
& D^{M}\left(X_{o}^{q}, Y_{o}^{q} ; d^{x}, d^{y}\right)=\max \sum_{g=1}^{G} \alpha^{g} \\
& \text { s.t. } \quad \beta^{g} \sum_{j=1}^{\delta_{g}} \lambda_{j}^{g} x_{i j}^{g} \leq x_{i o}^{q} \beta^{g}-\alpha^{g} d_{i}^{x}, \quad \forall g, i \\
& \beta^{g} \sum_{j=1}^{\delta_{g}} \lambda_{j}^{g} y_{r j}^{g} \geq y_{r o}^{q} \beta^{g}+\alpha^{g} d_{r}^{y}, \quad \forall g, r \\
& \beta^{g} \sum_{j=1}^{\delta_{g}} \lambda_{j}^{g}=\beta^{g}, \quad \forall g \\
& \sum_{g=1}^{G} \beta^{g}=1 \text {, } \\
& \beta^{g} \in\{0,1\}, \lambda_{j}^{g} \geq 0, \alpha^{g} \text { free in sign, } \quad \forall g, j .
\end{aligned}
$$

Taking into account that variables $\beta^{g}$ are binary, equality (15.5) implies that, in any feasible solution of program (15), exactly one variable $\beta^{g^{*}}=1$ and, therefore, $\beta^{g}=0$, for all $g \neq g^{*}$. Then 
(15.2) and (15.3) imply $\alpha^{g}=0$, for all $g \neq g^{*} \cdot{ }^{13}$ Therefore, for the fixed $g=g^{*}$, program (15) becomes program (14). Taking into account Remark 6, we have

$$
D^{M}\left(X_{o}^{q}, Y_{o}^{q} ; d^{x}, d^{y}\right)=\max \left\{D^{g}\left(X_{o}^{q}, Y_{o}^{q} ; d^{x}, d^{y}\right) \mid\left(X_{o}^{q}, Y_{o}^{q}\right) \in T^{g}, g \in \mathcal{G}\right\},
$$

which confirms that $D^{M}\left(X_{o}^{q}, Y_{o}^{q} ; d^{x}, d^{y}\right)$ is the directional distance function (13) evaluated in the metatechnology $T^{M}$.

Following a similar approach as in Section 3 based on the transformation of variables $\tilde{\lambda}_{j}^{g}=\beta^{g} \lambda_{j}^{g}$, for all $g \in \mathcal{G}$ and $j=1, \ldots, n$, we linearize program (15) as follows:

$$
\begin{aligned}
& \hat{D}^{M}\left(X_{o}^{q}, Y_{o}^{q} ; d^{x}, d^{y}\right)=\max \sum_{g=1}^{G} \alpha^{g} \\
& \text { s.t. } \quad \sum_{j=1}^{\delta_{g}} \lambda_{j}^{g} x_{i j}^{g} \leq x_{i o}^{q} \beta^{g}-\alpha^{g} d_{i}^{x}, \quad \forall g, i \\
& \sum_{j=1}^{\delta_{g}} \lambda_{j}^{g} y_{r j}^{g} \geq y_{r o}^{q} \beta^{g}+\alpha^{g} d_{r}^{y}, \quad \forall g, r \\
& \sum_{j=1}^{\delta_{g}} \lambda_{j}^{g}=\beta^{g}, \quad \forall g \\
& \sum_{g=1}^{G} \beta^{g}=1 \text {, } \\
& \beta^{g} \geq 0, \lambda_{j}^{g} \geq 0, \alpha^{g} \text { free in sign, } \quad \forall g, j .
\end{aligned}
$$

The equivalence of programs (15) and (16) and relationship between their optimal solutions can be established in the same way as the equivalence of programs (4) and (5) proved in Section 3. To avoid repetition, below we prove only the main result confirming the equivalence of programs (15) and (16). Similar to Definitions 2 and 3, we refer to $\hat{\Gamma}=\left\langle\hat{\alpha}, \hat{\beta}, \hat{\lambda}^{g} \mid g \in \mathcal{G}\right\rangle$ as a proper optimal solution to program (15) if $\hat{\beta}^{g}=0$ implies $\hat{\lambda}^{g}=0$, for all $g \in \mathcal{G}$. We refer to $\hat{\Gamma}$ as an elementary optimal solution to program (16) corresponding to group $g^{*} \in \mathcal{G}$, if vector $\tilde{\beta}$ has a single strictly positive component $\tilde{\beta}^{g^{*}}=1$.

Theorem 7. The optimal values of programs (15) and (16) are equal, i.e., $\hat{D}^{M}\left(X_{o}^{q}, Y_{o}^{q} ; d^{x}, d^{y}\right)=D^{M}\left(X_{o}^{q}, Y_{o}^{q} ; d^{x}, d^{y}\right)$. Any proper optimal solution $\hat{\Gamma}$ of program (15) is an elementary optimal solution of program (16) and vice versa, any elementary optimal solution of program (16) is a proper optimal solution of program (15).

The above linearization approach is applicable to the general definition of the directional distance function (13) with an arbitrary direction $\left(d^{x}, d^{y}\right) \neq 0$. In the special case when $d^{x}=X_{o}^{q}$ and

\footnotetext{
${ }^{13}$ Let $\beta^{g}=0$. If $d^{x} \neq 0$ then (15.2) implies $\alpha^{g}=0$. If $d^{y} \neq 0$ then (15.3) implies $\alpha^{g}=0$.
} 
$d^{y}=0$, program (16) becomes equivalent to program (5) that evaluates the input radial efficiency of $\operatorname{DMU}\left(X_{o}^{q}, Y_{o}^{q}\right)$ in the metatechnology $T^{M} \cdot{ }^{14}$ Similarly, the case $d^{x}=0$ and $d^{y}=Y_{o}^{q}$ results in program (16) leading to the assessment of the output radial efficiency of DMU $\left(X_{o}^{q}, Y_{o}^{q}\right)$.

It is straightforward to modify the linear program (16) to the cases of CRS, NIRS and NDRS, by removing or modifying the normalizing equality (16.4). This is similar to the case of input radial efficiency discussed in Remark 2.

\section{A computational example}

To illustrate the calculation of one-sided scale elasticities and evaluation of RTS, consider the data set in Table 3. It contains 32 DMUs that belong to four group technologies: group 1 (9 DMUs), group 2 (12 DMUs), group 3 (11 DMUs) and group 4 (8 DMUs). Note that six of these DMUs are members of more than one technology. Each DMU is characterized by three inputs and two outputs.

\begin{tabular}{|c|c|c|c|c|c|c|c|c|c|c|c|c|c|}
\hline & & \multicolumn{3}{|c|}{ Input } & \multicolumn{2}{c|}{ Output } & & \multicolumn{3}{c|}{ Input } & \multicolumn{2}{c|}{ Output } \\
\hline DMU & Group & I1 & I2 & I3 & O1 & O2 & DMU & Group & I1 & I2 & I3 & O1 & O2 \\
\hline 1 & 1 & 5.75 & 8.63 & 7.19 & 9.05 & 16.41 & 17 & 2 & 9.49 & 13.27 & 11.24 & 9.07 & 17.06 \\
\hline 2 & 1 & 5.50 & 7.20 & 6.00 & 7.00 & 13.45 & 18 & 2,3 & 22.20 & 32.00 & 28.10 & 6.50 & 13.00 \\
\hline 3 & 1 & 4.00 & 6.00 & 5.00 & 8.00 & 15.00 & 19 & 3 & 17.66 & 26.49 & 22.08 & 19.33 & 36.25 \\
\hline 4 & 1 & 14.00 & 8.00 & 8.10 & 6.00 & 14.00 & 20 & 3 & 20.10 & 27.00 & 28.30 & 18.80 & 35.00 \\
\hline 5 & 1 & 7.50 & 11.25 & 9.38 & 10.10 & 17.81 & 21 & 3 & 18.20 & 31.10 & 25.00 & 19.90 & 33.00 \\
\hline 6 & 1 & 5.38 & 7.81 & 7.49 & 7.03 & 14.26 & 22 & 3 & 17.00 & 25.49 & 21.25 & 20.00 & 37.50 \\
\hline 7 & 1,2 & 11.00 & 16.50 & 13.75 & 11.00 & 20.63 & 23 & 3 & 23.00 & 27.40 & 30.00 & 15.00 & 30.34 \\
\hline 8 & $1,2,3$ & 24.50 & 33.12 & 31.00 & 4.80 & 9.54 & 24 & 3 & 20.60 & 29.25 & 27.50 & 17.45 & 31.67 \\
\hline 9 & 1,2 & 16.10 & 23.40 & 19.45 & 7.00 & 14.10 & 25 & 3 & 20.33 & 26.95 & 26.04 & 17.17 & 33.29 \\
\hline 10 & 2 & 15.70 & 20.00 & 18.00 & 10.25 & 20.40 & 26 & 3,4 & 21.00 & 31.49 & 26.24 & 22.00 & 41.25 \\
\hline 11 & $2,3,4$ & 15.00 & 22.50 & 18.75 & 16.00 & 30.00 & 27 & 4 & 27.00 & 40.49 & 33.75 & 26.00 & 48.75 \\
\hline 12 & 2 & 15.19 & 20.49 & 18.09 & 12.62 & 21.98 & 28 & 4 & 24.00 & 35.99 & 29.99 & 21.00 & 41.25 \\
\hline 13 & 2 & 12.97 & 19.47 & 16.22 & 13.50 & 25.31 & 29 & 4 & 29.00 & 38.00 & 32.00 & 20.50 & 38.98 \\
\hline 14 & 2 & 11.98 & 17.98 & 14.98 & 12.25 & 22.97 & 30 & 4 & 26.20 & 40.10 & 32.00 & 20.00 & 37.80 \\
\hline 15 & 2 & 17.70 & 21.34 & 19.35 & 12.85 & 22.55 & 31 & 4 & 26.40 & 38.39 & 32.55 & 19.38 & 38.35 \\
\hline 16 & 2 & 13.98 & 20.98 & 17.48 & 14.75 & 27.66 & 32 & 4 & 18.40 & 25.00 & 21.48 & 16.30 & 33.75 \\
\hline
\end{tabular}

Table 3. The data set for four groups of DMUs with three inputs (I1, I2 and I3) and two outputs (O1 and O2).

To be specific, consider assessing the input radial meta-efficiency of these DMUs under the assumption of VRS, either by the envelopment model (5) or its dual multiplier model (6). Table 4 shows the resulting efficiency scores.

\footnotetext{
${ }^{14}$ In this case, we denote $\theta^{g}=\beta^{g}-\alpha^{g}$ and restate the right-hand side of constraints (16.2) as $\theta^{g} x_{i o}^{q}$, for all $g$ and $i$. Then maximizing the objective function in (16.1) is equivalent to minimising $1-\sum_{g=1}^{G} \alpha^{g}=\sum_{g=1}^{G} \beta^{g}-\sum_{g=1}^{G} \alpha^{g}=\sum_{g=1}^{G} \theta^{g}$, where the first equality follows from (16.5).
} 


\begin{tabular}{|c|c|c|c|c|c|c|c|}
\hline DMU & $\widehat{E f f}^{M}()$. & DMU & $\widehat{E f f}^{M}()$. & DMU & $\widehat{E f f}^{M}()$. & DMU & $\widehat{E f f}^{M}()$. \\
\hline 1 & 1.000 & 9 & 0.257 & 17 & 0.742 & 25 & 0.884 \\
\hline 2 & 0.833 & 10 & 0.783 & 18 & 0.188 & 26 & 1.000 \\
\hline 3 & 1.000 & 11 & 1.000 & 19 & 0.944 & 27 & 1.000 \\
\hline 4 & 0.750 & 12 & 0.878 & 20 & 0.911 & 28 & 0.875 \\
\hline 5 & 1.000 & 13 & 1.000 & 21 & 0.931 & 29 & 0.733 \\
\hline 6 & 0.768 & 14 & 1.000 & 22 & 1.000 & 30 & 0.677 \\
\hline 7 & 1.000 & 15 & 0.858 & 23 & 0.826 & 31 & 0.699 \\
\hline 8 & 0.181 & 16 & 1.000 & 24 & 0.806 & 32 & 0.960 \\
\hline
\end{tabular}

Table 4. The input radial meta-efficiency of DMUs.

Note that 11 DMUs are input radial efficient against the metafrontier. ${ }^{15}$ According to Theorem 6, the one-sided scale elasticities $\varepsilon_{o}^{M-}$ and $\varepsilon_{o}^{M+}$ at each of these DMUs are computed by evaluating the one-sided scale elasticities $\varepsilon_{o}^{g-}$ and $\varepsilon_{o}^{g+}$, separately for each of its active group frontiers $g \in \mathcal{G}^{0}$, and then by taking their minimum and maximum, respectively. The set of all active group frontiers $\mathcal{G}^{0}$ can be found by solving program (8). Table 5 summarizes the results.

\begin{tabular}{|c|c|c|c|c|}
\hline DMU & $\begin{array}{c}\text { Active group } \\
\text { frontiers }\end{array}$ & $\varepsilon_{0}^{M-}$ & $\varepsilon_{0}^{M+}$ & Type of RTS \\
\hline 1 & 1 & 0.381 & 0.281 & DRS \\
\hline 3 & 1 & $+\infty$ & 0.214 & CRS \\
\hline 5 & 1 & 0.446 & 0.191 & DRS \\
\hline 7 & 1,2 & 0.429 & 1.275 & DRS-IRS \\
\hline 11 & $2,3,4$ & 1.150 & 1.875 & IRS-IRS \\
\hline 13 & 2 & 1.213 & 1.189 & IRS \\
\hline 14 & 2 & 1.247 & 1.233 & IRS \\
\hline 16 & 2 & 1.176 & 1.159 & IRS \\
\hline 22 & 3 & 1.705 & 0.425 & CRS \\
\hline 26 & 3,4 & 0.478 & 0.635 & DRS-DRS \\
\hline 27 & 4 & 0.692 & 0.000 & DRS \\
\hline
\end{tabular}

Table 5. Active group frontiers of the efficient DMUs, their one-sided scale elasticities $\varepsilon_{0}^{M-}$ and $\varepsilon_{0}^{M+}$, and the type of RTS evaluated on the metafrontier.

As can be seen from Table 5, all but three DMUs are located on a single group frontier. According to Corollary 3, for these DMUs, the interpretation of one-sided scale elasticities $\varepsilon_{o}^{M-}$ and $\varepsilon_{o}^{M+}$ evaluated on the metafrontier is similar to the case of a single VRS technology. For example,

\footnotetext{
${ }^{15}$ These DMUs are also output-radial efficient in the metatechnology and thus satisfy the assumption stated in Footnote 11.
} 
for $\mathrm{DMU}_{1}$ (for which the only active frontier is that of group 1 to which $\mathrm{DMU}_{1}$ belongs) we have $\varepsilon_{1}^{M-}=0.381$ and $\varepsilon_{1}^{M+}=0.281$. Because $\varepsilon_{1}^{M+} \leq \varepsilon_{1}^{M-}<1, \mathrm{DMU}_{1}$ exhibits DRS on the metafrontier. This implies that, if the input vector of this DMU is increased in a marginally small proportion, e.g., by $1 \%$, its output vector would increase in a smaller proportion, by $0.281 \%$. If its input vector is reduced by $1 \%$, the output vector would decrease by $0.381 \%$.

In contrast, for those DMUs which are located at the intersection of more than one group frontier, the inequality (12) for the one-sided scale elasticities $\varepsilon_{o}^{M-}$ and $\varepsilon_{o}^{M+}$ evaluated on the metafrontier may not hold. Hence, the interpretation of the one-sided scale elasticities requires a more careful treatment that goes beyond the standard definition of RTS.

For example, for $\mathrm{DMU}_{11}$ (which is a member of groups 2, 3 and 4) we have $\varepsilon_{11}^{M-}=1.150$ and $\varepsilon_{11}^{M+}=1.875$. For this DMU, we have $\varepsilon_{11}^{M-}>1$ and $\varepsilon_{11}^{M+}>1$. This means that that $\mathrm{DMU}_{11}$ exhibits IRS for any marginal change (increase or decrease) of its input vector. However, because $\varepsilon_{11}^{M-}<\varepsilon_{11}^{M+}$, the IRS characterization is stronger (identified by the higher scale elasticity) on the right of $\mathrm{DMU}_{11}$ than on the left. For example, the right-hand scale elasticity $\varepsilon_{11}^{M+}=1.875$ is equal to the right-hand scale elasticity $\varepsilon_{11}^{G 3+}=1.875$, which is evaluated at this DMU on the frontier of the technology $T^{3}$, i.e. $\varepsilon_{11}^{M+}=\max \left\{\varepsilon_{11}^{G 2+}=0, \varepsilon_{11}^{G 3+}=1.875, \varepsilon_{11}^{G 4+}=0.938\right\}$. This suggests that the highest increase of the output vector of $\mathrm{DMU}_{11}$ is achieved if $\mathrm{DMU}_{11}$ stays in technology $T^{3}$ and moves along its efficient frontier. Similarly, $\mathrm{DMU}_{26}$ exhibits DRS both on the right and on the left (i.e., in the increasing and decreasing scenarios). However, in contrast with the standard case of a single VRS technology, the DRS characterization of $\mathrm{DMU}_{26}$ is stronger on the left (as identified by the lower scale elasticity) than on the right.

Another nonstandard situation can be seen where $\varepsilon_{0}^{M-}<1$ and $\varepsilon_{0}^{M+}>1$. Using $\mathrm{DMU}_{7}$ (which is a member of groups 1 and 2) as an example, we have $\varepsilon_{7}^{M-}=0.429<1$ and $\varepsilon_{7}^{M+}=1.275>1$. Therefore, this DMU exhibits DRS on the left and IRS on the right, which is impossible in the single VRS technology. Details of our computations show that the right-hand scale elasticity $\varepsilon_{7}^{M+}=1.275$ is equal to the right-hand scale elasticity $\varepsilon_{7}^{G 2+}=1.275$, i.e. $\varepsilon_{7}^{M+}=\max \left\{\varepsilon_{7}^{G 1+}=0, \varepsilon_{7}^{G 2+}=1.275\right\}$. The left-hand scale elasticity $\varepsilon_{7}^{M-}=0.429$ is, however, equal to the left-hand scale elasticity $\varepsilon_{7}^{G 1-}=0.429$, i.e. $\varepsilon_{7}^{M-}=\min \left\{\varepsilon_{7}^{G 1-}=0.429, \varepsilon_{7}^{G 2-}=1.278\right\}$. This implies that increasing the input vector of $\mathrm{DMU}_{7}$ by $1 \%$ leads to a maximum increase of the output vector by $\varepsilon_{7}^{M+}=1.275 \%$ if this DMU stays within technology $T^{2}$ and moves along its efficient frontier. However, reducing the input vector of $\mathrm{DMU}_{7}$ by $1 \%$ would lead to the reduction of its maximum possible output by $\varepsilon_{7}^{M-}=0.429 \%$ provided that $\mathrm{DMU}_{7}$ stays within technology $T^{1}$ and moves along its efficient frontier. 


\section{Conclusion}

The metafrontier approach to efficiency assessment of DMUs that belong to different group technologies is well-established in DEA. According to this approach, the efficiency of each DMU is assessed with respect to all DMUs in the same group, and also with respect to the metatechnology that includes all DMUs in all group technologies. As noted by O'Donnell et al. (2008), the gap between the efficiency of a DMU in its group technology and its efficiency in the metatechnology is interpretable as a measure of potential performance improvement of the DMU, provided it is possible to change the environment in which it operates.

In practical applications, individual group technologies are often assumed to be convex sets and are modelled by the conventional, usually VRS, DEA models. However, the metatechnology may not always be assumed to be a convex set. In this case, assessing the efficiency of a DMU against the metafrontier requires either implementing an enumeration algorithm and solving a linear program at each of its steps, or solving an equivalent mixed integer linear program.

In our paper we show that the task of evaluating the efficiency of a DMU in a nonconvex metatechnology can be accomplished by solving a single linear program, both in the primal (envelopment) and dual (multiplier) forms. We show that our method is applicable in the cases in which group technologies satisfy different returns-to-scale assumptions, including VRS and CRS, and also in both the input and output orientations. Our main development concerns the task of assessing the input and output radial efficiency of DMUs, but we subsequently extend our approach to the more general framework of directional distance functions.

From a practical perspective, our approach offers the convenience of solving a single linear program for each DMU under the evaluation, which always has a finite optimal solution. We also show the usefulness of the programs stated in the dual space for the RTS characterization of efficient DMUs on the metafrontier.

Our development opens up further research avenues. In particular, it is worth considering a similar linearizing approach for technologies other than the conventional VRS or CRS technologies. This includes technologies with weakly disposable bad outputs (Kuosmanen, 2005; Kuosmanen and Podinovski, 2009), the hybrid RTS technology of Podinovski (2004a), a similar in spirit FDH technology of Afsharian et al. (2015), and technologies expanded by the specification of production trade-offs (Podinovski, 2004b). Furthermore, it would be useful to develop a general linearizing approach applicable to any polyhedral DEA technology defined by Podinovski et al. (2016), of which most of the above examples are special cases.

\section{Acknowledgment}

The financial support of the Deutsche Forschungsgemeinschaft (DFG) in the context of the research fund AH 90/5-1 is gratefully acknowledged. 


\section{Appendix: Proofs}

Lemma 1. Let $\tilde{\Gamma}=\left\langle\tilde{\theta}, \tilde{\beta}, \tilde{\lambda}^{g} \mid g \in \mathcal{G}\right\rangle$ be any optimal solution of program (5). Then, for any $g \in \mathcal{G}$, $\tilde{\beta}^{g}=0$ implies $\tilde{\theta}^{g}=0$ and $\tilde{\lambda}_{j}^{g}=0$, for all $j=1, \ldots, \delta_{g}$.

Proof of Lemma 1. By (5.2), $\tilde{\theta}^{g} \geq 0$. Treating $\beta^{g}=\tilde{\beta}^{g}$ as a constant, $\tilde{\theta}^{g}$ minimizes $\theta^{g}$, subject to constraints (5.2) - (5.4). If $\tilde{\beta}^{g}=0$ then the values $\theta^{g}=0$ and $\lambda_{j}^{g}=0$, for all $j=1, \ldots, \delta_{g}$, satisfy constraints (5.2) - (5.4). Therefore, $\tilde{\theta}^{g}=0$, and (5.4) implies $\tilde{\lambda}_{j}^{g}=0$, for all $j=1, \ldots, \delta_{g}$. Note that this implication can also be proved without a reference to equality (5.4), e.g., under the assumption of CRS for which (5.4) is missing from (5). Indeed, because $\tilde{\theta}^{g}=0$, the left-hand side of (5.2) is a zero vector. Because each DMU $j=1, \ldots, \delta_{g}$ has at least one positive input $x_{i j}^{g}>0$, we have $\lambda_{j}^{g}=0$, for all $j=1, \ldots, \delta_{g}$.

Lemma 2. Let $\tilde{\Gamma}=\left\langle\tilde{\theta}, \tilde{\beta}, \tilde{\lambda}^{g} \mid g \in \mathcal{G}\right\rangle \quad$ be any optimal solution of program (5). Then $\tilde{\theta}^{g_{1}} / \tilde{\beta}^{g_{1}}=\tilde{\theta}^{g_{2}} / \tilde{\beta}^{g_{2}}$, for any $g_{1}, g_{2} \in \mathcal{G}^{*}=\left\{g \mid \tilde{\beta}^{g}>0, g \in \mathcal{G}\right\}$.

Proof of Lemma 2. Assume that Lemma 2 is not true. Then there exist $g_{1}, g_{2} \in \mathcal{G}^{*}$ such that $\tilde{\theta}^{g_{1}} / \tilde{\beta}^{g_{1}}>\tilde{\boldsymbol{\theta}}^{g_{2}} / \tilde{\boldsymbol{\beta}}^{g_{2}}$. Then $\tilde{\boldsymbol{\beta}}^{g_{2}} \tilde{\boldsymbol{\theta}}^{g_{1}}>\tilde{\boldsymbol{\beta}}^{g_{1}} \tilde{\boldsymbol{\theta}}^{g_{2}}$. Let us show that solution $\tilde{\Gamma}$ can be improved and therefore is not optimal. Indeed, define vector $\hat{\beta}^{g}$ as follows. For $g=g_{1}$, define $\hat{\beta}^{g_{1}}=\hat{\theta}^{g_{1}}=0$ and let $\hat{\lambda}_{j}^{g_{1}}=0$ for all $j=1, \ldots, \delta_{g_{1}}$. Define $\hat{\beta}^{g_{2}}=\tilde{\beta}^{g_{1}}+\tilde{\beta}^{g_{2}}$. This increases $\beta^{g_{2}}$ from the value $\tilde{\beta}^{g_{2}}$ in solution $\tilde{\Gamma}$ to $\hat{\beta}^{g_{2}}$, i.e., by the factor $\varepsilon=\left(\tilde{\beta}^{g_{1}}+\tilde{\beta}^{g_{2}}\right) / \tilde{\beta}^{g_{2}}$. Define $\hat{\theta}^{g_{2}}=\varepsilon \tilde{\theta}^{g_{2}}$ and $\hat{\lambda}_{j}^{g_{2}}=\varepsilon \tilde{\lambda}_{j}^{g_{2}}$ for all $j=1, \ldots, \delta_{g_{2}}$. Finally, for all $g \neq g_{1}, g_{2}$, we leave the corresponding components of $\tilde{\Gamma}$ unchanged, i.e., we let $\hat{\beta}^{g}=\tilde{\beta}^{g}, \hat{\theta}^{g}=\tilde{\theta}^{g}$ and $\hat{\lambda}_{j}^{g}=\tilde{\lambda}_{j}^{g}$, for all $j=1, \ldots, \delta_{g}$.

It is straightforward to verify that the new solution $\hat{\Gamma}=\left\langle\hat{\theta}, \hat{\beta}, \hat{\lambda}^{g} \mid g \in \mathcal{G}\right\rangle$ is feasible in program (5). Comparing the objective function at solutions $\hat{\Gamma}$ and $\tilde{\Gamma}$, we have

$$
\sum_{g=1}^{G} \hat{\theta}^{g}-\sum_{g=1}^{G} \tilde{\theta}^{g}=\left(0+\frac{\tilde{\beta}^{g_{1}}+\tilde{\beta}^{g_{2}}}{\tilde{\beta}^{g_{2}}} \tilde{\theta}^{g_{2}}\right)-\left(\tilde{\theta}^{g_{1}}+\tilde{\theta}^{g_{2}}\right)=\frac{\tilde{\beta}^{g_{1}} \tilde{\theta}^{g_{2}}-\tilde{\beta}^{g_{2}} \tilde{\theta}^{g_{1}}}{\tilde{\beta}^{g_{2}}}<0 .
$$

Therefore, $\tilde{\Gamma}$ is not an optimal solution to program (5).

Proof of Theorem 1. Statement (i) of this theorem is proved as Lemma 1. To prove statement (ii), let $\tilde{\beta}^{g}>0$. Suppose $\left|\mathcal{G}^{*}\right|=1$, i.e., the set $\mathcal{G}^{*}$ consists of a single element $g^{*}$. Taking into account (5.5), 
$\tilde{\beta}^{g^{*}}=1$ and $\tilde{\beta}^{g}=0$, for all $g \neq g^{*}, g \in \mathcal{G}$. By Lemma $1, \tilde{\theta}^{g}=0$ for all $g \neq g^{*}, g \in \mathcal{G}$. Therefore, $\widehat{E f f}^{M}\left(X_{o}^{p}, Y_{o}^{p}\right)=\tilde{\theta}^{g^{*}}=\tilde{\theta}^{g^{*}} / \tilde{\beta}^{g^{*}}$.

Let $\left|\mathcal{G}^{*}\right|>1$. Consider any $g^{*} \in \mathcal{G}^{*}$. First, let us prove that there exists an alternative optimal solution $\hat{\Gamma}=\left\langle\hat{\theta}, \hat{\beta}, \hat{\lambda}^{g} \mid g \in \mathcal{G}\right\rangle$ such that the only positive component of vector $\hat{\beta}$ is $\hat{\beta}^{g^{*}}=1$. For $g=g^{*}$, define $\hat{\beta}^{g^{*}}=\sum_{g \in \mathcal{G}^{*}} \tilde{\beta}^{g}=1$. This increases the value of variable $\beta^{g^{*}}$ from $\tilde{\beta}^{g^{*}}$ in solution $\tilde{\Gamma}$ to $\hat{\beta}^{g^{*}}=1$, i.e., by the factor $\left(\sum_{g \in \mathcal{G}^{*}} \tilde{\beta}^{g}\right) / \tilde{\beta}^{g^{*}}=1 / \tilde{\beta}^{g^{*}}$. To keep all constraints satisfied, define $\hat{\theta}^{g^{*}}=\tilde{\theta}^{g^{*}} / \tilde{\beta}^{g^{*}}$ and $\hat{\lambda}_{j}^{g^{*}}=\tilde{\lambda}_{j}^{g^{*}} / \tilde{\beta}^{g^{*}}$ for all $j=1, \ldots, \delta_{g^{*}}$. For all $g \in \mathcal{G}^{*}$, define $\hat{\beta}^{g}=\hat{\theta}^{g}=0$ and let $\hat{\lambda}_{j}^{g}=0$ for all $j=1, \ldots, \delta_{g}$. For all $g \in \mathcal{G} \backslash \mathcal{G}^{*}$, we leave the corresponding components of $\tilde{\Gamma}$ unchanged, i.e., we let $\hat{\beta}^{g}=\tilde{\beta}^{g}=0, \hat{\theta}^{g}=\tilde{\theta}^{g}=0$ and, taking into account Lemma $1, \quad \hat{\lambda}_{j}^{g}=\tilde{\lambda}_{j}^{g}=0$, for all $j=1, \ldots, \delta_{g}$.

The new solution $\hat{\Gamma}=\left\langle\hat{\theta}, \hat{\beta}, \hat{\lambda}^{g} \mid g \in \mathcal{G}\right\rangle$ is feasible in (5). Calculating the difference between the values of the objective function at $\hat{\Gamma}$ and $\tilde{\Gamma}$, we have

$$
\sum_{g=1}^{G} \hat{\theta}^{g}-\sum_{g=1}^{G} \tilde{\theta}^{g}=\hat{\theta}^{g}-\sum_{g \in \mathcal{G}^{*}} \tilde{\theta}^{g}=\frac{\sum_{g \in \mathcal{G}^{\mathcal{G}^{*}}} \tilde{\beta}^{g}}{\tilde{\beta}^{g^{*}}} \tilde{\theta}^{g^{*}}-\sum_{g \in \mathcal{G}^{*}} \tilde{\theta}^{g}=\frac{\sum_{g \in \mathcal{G}^{*}}\left(\tilde{\beta}^{g} \tilde{\theta}^{g^{*}}-\tilde{\beta}^{g^{*}} \tilde{\theta}^{g}\right)}{\tilde{\beta}^{g^{*}}}=0,
$$

where the last equality follows from Lemma 2, which implies that $\tilde{\beta}^{g} \tilde{\theta}^{g^{*}}=\tilde{\beta}^{g^{*}} \tilde{\theta}^{g}$, for all $g \in \mathcal{G}^{*}$. Therefore, solution $\hat{\Gamma}$ is optimal in program (5). Because the only positive component of vector $\hat{\beta}$ is $\hat{\beta}^{g^{*}}=1$, we have $\hat{\theta}^{g^{*}}=\widehat{E f f}^{M}\left(X_{o}^{q}, Y_{o}^{q}\right)$. Finally, by the definition of $\hat{\beta}^{g^{*}}$ and $\hat{\theta}^{g^{*}}$, and by Lemma 2 , for all $g \in \mathcal{G}^{*}$, we have

$$
\tilde{\theta}^{g} / \tilde{\beta}^{g}=\tilde{\theta}^{g^{*}} / \tilde{\beta}^{g^{*}}=\hat{\theta}^{g^{*}} / \hat{\beta}^{g^{*}}=\hat{\theta}^{g^{*}} / 1=\widehat{E f f}^{M}\left(X_{o}^{q}, Y_{o}^{q}\right) .
$$

Lemma 3. The optimal value of program (5) is equal to the optimal value of program (4), i.e., $\operatorname{Eff}^{M}\left(X_{p}^{q}, Y_{p}^{q}\right)=\widehat{\operatorname{Eff}}^{M}\left(X_{o}^{q}, Y_{o}^{q}\right)$.

Proof of Lemma 3. It suffices to prove the following two statements:

(i) For any feasible solution $\Gamma$ of program (4) there exists a feasible solution $\tilde{\Gamma}$ of program (5) such that, for these solutions, the objective functions of programs (4) and (5) are equal.

(ii) For any optimal solution $\Gamma$ of program (5) there exists a feasible solution $\hat{\Gamma}$ of program (4) for which the objective functions of programs (5) and (4) are equal. 
To prove (i), let $\Gamma=\left\langle\theta, \beta, \lambda^{g} \mid g \in \mathcal{G}\right\rangle$ be a feasible solution to program (4). Define vectors $\tilde{\lambda}^{g}=\beta^{g} \lambda^{g}$, for all $g \in \mathcal{G}$. Then $\tilde{\Gamma}=\left\langle\theta, \beta, \tilde{\lambda}^{g} \mid g \in \mathcal{G}\right\rangle$ is feasible in (5). The objective functions of both programs for these solutions are equal.

To prove (ii), let $\Gamma=\left\langle\theta, \beta, \lambda^{g} \mid g \in \mathcal{G}\right\rangle$ be an optimal solution to program (5). Select any $g^{*} \in \mathcal{G}^{*}=\left\{g \in \mathcal{G} \mid \beta^{g}>0\right\}$. Define a feasible solution $\hat{\Gamma}$ to program (4) as follows. For $g=g^{*}$, let $\hat{\beta}^{g^{*}}=1, \hat{\theta}^{g^{*}}=\theta^{g^{*}} / \beta^{g^{*}}$, and define vector $\hat{\lambda}^{g^{*}}=\lambda^{g^{*}} / \beta^{g^{*}}$. For all $g \in \mathcal{G}, g \neq g^{*}$, let $\hat{\beta}^{g}=\hat{\theta}^{g}=0$, and let $\hat{\lambda}^{g}$ be zero vectors. Then $\hat{\Gamma}=\left\langle\hat{\theta}, \hat{\beta}, \hat{\lambda}^{g} \mid g \in \mathcal{G}\right\rangle$ is a feasible solution to program (4). For this solution, the objective function of program (4) is equal to $\hat{\theta}^{g^{*}}=\theta^{g^{*}} / \beta^{g^{*}}$. By Theorem 1, this is equal to the optimal value of program (5).

Proof of Corollary 1. By Definition 3, for the vector $\tilde{\beta}$ in the elementary optimal solution $\tilde{\Gamma}$ we have $\tilde{\beta}^{g^{*}}=1$. Then statement (ii) of Theorem 1 implies $\tilde{\theta}^{g^{*}}=\widehat{E f f}^{M}\left(X_{o}^{q}, Y_{o}^{q}\right)$. Furthermore, by statement (i) of Theorem 1, we have $\tilde{\lambda}_{j}^{g}=0$, for all $j=1, \ldots, \delta_{g}$ and all $g \in \mathcal{G}, g \neq g^{*}$. Therefore, from (5.2), $\tilde{\theta}^{g}=0$, for all $g \in \mathcal{G}, g \neq g^{*}$.

Proof of Theorem 2. Statement (i) is proved as Lemma 3. Statements (ii) and (iii) are elementary. In particular, the proof of statement (iii) uses Lemma 1. Let us prove statement (iv). Let $\tilde{\Gamma}=\left\langle\tilde{\theta}, \tilde{\beta}, \tilde{\lambda}^{g} \mid g \in \mathcal{G}\right\rangle$ be any optimal solution to program (5), and let $\mathcal{G}^{*}=\left\{g \mid \tilde{\beta}^{g}>0, g \in \mathcal{G}\right\}$. It suffices to consider the case $\left|\mathcal{G}^{*}\right|>1$. In this case, by Lemma 1 , all vectors $\tilde{\lambda}^{g}, g \in \mathcal{G} \backslash \mathcal{G}^{*}$, are zero vectors.

Repeating the proof of Theorem 1 for the case $\left|\mathcal{G}^{*}\right|>1$, we establish that there exists an elementary optimal solution $\Gamma_{g}$ to (5) corresponding to each group $g \in \mathcal{G}^{*}$. (In the proof of Theorem $1, \Gamma_{g}$ is denoted $\hat{\Gamma}=\left\langle\hat{\theta}, \hat{\beta}, \hat{\lambda}^{g} \mid g \in \mathcal{G}\right\rangle$.) By the definition of components of vectors $\hat{\theta}, \hat{\beta}$ and $\hat{\lambda}^{g}$, $g \in \mathcal{G}$, in the proof of Theorem $1, \tilde{\Gamma}$ is the convex combination of solutions $\Gamma_{g}$ taken with the weights $\tilde{\beta}^{g}, g \in \mathcal{G}^{*}$.

Proof of Corollary 2. By statement (iv) of Theorem 2, any optimal solution $\tilde{\Gamma}$ of program (5) is a convex combination of a finite number of elementary optimal solutions $\Gamma_{g}$ of program (5). By statement (iii) of Theorem 2, each such elementary optimal solution is a proper optimal solution of program (4). Therefore, $\tilde{\mathcal{S}} \subseteq$ conv $\hat{\mathcal{S}}$. Conversely, let $\hat{\Gamma} \in \operatorname{conv} \hat{\mathcal{S}}$. By Carathéodory's theorem, $\hat{\Gamma}$ is a convex combination of a finite number of proper optimal solutions $\Gamma_{k} \in \hat{\mathcal{S}}, k=1, \ldots, K$, of program 
(4). By statement (ii) of Theorem 2, every such $\Gamma_{k}$ is optimal in (5). Because (5) is a linear program, the set of its optimal solutions is convex. Therefore, $\hat{\Gamma} \in \tilde{\mathcal{S}}$, and conv $\hat{\mathcal{S}} \subseteq \tilde{\mathcal{S}}$.

Proof of Theorem 3. For any $g \in \mathcal{G}$, because $\Delta=\left\langle\tilde{u}^{g}, \tilde{v}^{g}, \tilde{\omega}^{g}, \tilde{\varphi} \mid g \in \mathcal{G}\right\rangle$ is a feasible solution of (6), $\left\langle\tilde{u}^{g}, \tilde{v}^{g}, \tilde{\omega}^{g}\right\rangle$ is feasible in (7). Assume that there exists a $g \in \mathcal{G}^{0}$ such that the corresponding inequality (6.2) is strict. Then, for the solution $\left\langle\tilde{u}^{g}, \tilde{v}^{g}, \tilde{\omega}^{g}\right\rangle$, the objective function of (7) is strictly greater than $\tilde{\varphi}=E f f^{M}\left(X_{o}^{q}, Y_{o}^{q}\right)$, which contradicts the definition of the set $\mathcal{G}^{0}$. Therefore, for all $g \in \mathcal{G}^{0}$, the inequalities (6.2) are satisfied as equalities, and the first statement of Theorem 1 follows.

To prove the second statement, consider any set $\mathcal{G}^{*}$ such that $\mathcal{G}^{0} \subseteq \mathcal{G}^{*} \subseteq \mathcal{G}$. Define the optimal solution $\Delta^{*}$ as follows. For each $g \in \mathcal{G}^{0}$, let $\left\langle\hat{u}^{g}, \hat{v}^{g}, \hat{\omega}^{g}\right\rangle$ be any optimal solution to the corresponding program (7). By the definition of the set $\mathcal{G}^{0}$, for all $g \in \mathcal{G}^{0}$, we have $\operatorname{Eff}^{g}\left(X_{o}^{q}, Y_{o}^{q}\right)=\operatorname{Eff}^{M}\left(X_{o}^{q}, Y_{o}^{q}\right)$. Denote $\hat{\varphi}=\operatorname{Eff}^{M}\left(X_{o}^{q}, Y_{o}^{q}\right)$.

Now let $g \in \mathcal{G} \backslash \mathcal{G}^{0}$. Then the optimal value of the corresponding program (7) is either strictly greater than $\hat{\varphi}$ or it is unbounded. Therefore, there exists a feasible solution $\left\langle\hat{u}^{g}, \hat{v}^{g}, \hat{\omega}^{g}\right\rangle$ of program (7) for which its objective function is $\hat{\varepsilon}^{g}=\sum_{r=1}^{s} \hat{u}_{r}^{g} y_{r o}^{q}+\hat{\omega}^{g}>\hat{\varphi}$. If $g \in \mathcal{G}^{*} \backslash \mathcal{G}^{0}$, we further define $\left\langle\bar{u}^{g}, \bar{v}^{g}, \bar{\omega}^{g}\right\rangle=\left\langle\left(\hat{\varphi} / \hat{\varepsilon}^{g}\right) \hat{u}^{g}, \hat{v}^{g},\left(\hat{\varphi} / \hat{\varepsilon}^{g}\right) \hat{\omega}^{g}\right\rangle$. Because $\hat{\varphi} / \hat{\varepsilon}^{g}<1$, the solution $\left\langle\bar{u}^{g}, \bar{v}^{g}, \bar{\omega}^{g}\right\rangle$ is feasible in program (7), and the corresponding objective function of (7) is equal to $\hat{\varphi}$. This implies that the solution $\left\langle\bar{u}^{g}, \bar{v}^{g}, \bar{\omega}^{g}\right\rangle$ satisfies (6.2) as equality. Finally, if $g \in \mathcal{G} \backslash \mathcal{G}^{*}$, we keep the solution $\left\langle\hat{u}^{g}, \hat{v}^{g}, \hat{\omega}^{g}\right\rangle$ unchanged.

We now consider the following solution $\Delta^{*}=\left\langle u^{g}, v^{g}, \omega^{g}, \varphi \mid g \in \mathcal{G}\right\rangle$. Let $\varphi=\hat{\varphi}$ be as defined above. For all $g \in \mathcal{G}^{0}$ and $g \in \mathcal{G} \backslash \mathcal{G}^{*}$, we take $\left\langle u^{g}, v^{g}, \omega^{g}\right\rangle=\left\langle\hat{u}^{g}, \hat{v}^{g}, \hat{\omega}^{g}\right\rangle$. For all $g \in \mathcal{G}^{*} \backslash \mathcal{G}^{0}$, we take $\left\langle u^{g}, v^{g}, \omega^{g}\right\rangle=\left\langle\bar{u}^{g}, \bar{v}^{g}, \bar{\omega}^{g}\right\rangle$. Then $\Delta^{*}$ is feasible in program (6), and the corresponding objective function of (6) is equal to $\hat{\varphi}=E f f^{M}\left(X_{o}^{q}, Y_{o}^{q}\right)$. Therefore, $\Delta^{*}$ is optimal in program (6). We finally note that, for the solution $\Delta^{*}$, the constraints (6.2) are satisfied as equalities for all $g \in \mathcal{G}^{0} \cup\left(\mathcal{G}^{*} \backslash \mathcal{G}^{0}\right)=\mathcal{G}^{*}$, and (6.2) are strict inequalities for $g \in \mathcal{G} \backslash \mathcal{G}^{*}$. Therefore, $\mathcal{G}\left(\Delta^{*}\right)=\mathcal{G}^{*}$.

Proof of Theorem 4. By Theorem 5 proved below we have $\tilde{\varphi}=E f f{ }^{M}\left(X_{o}^{q}, Y_{o}^{q}\right)$. Because solution $\tilde{\tilde{\Delta}}$ is feasible in program (8), and by formula (9), $\tilde{\tilde{\Delta}}$ is a feasible solution of program (6), for which its 
objective function is equal to $\tilde{\varphi}=E f f^{M}\left(X_{o}^{q}, Y_{o}^{q}\right)$. Therefore, $\tilde{\tilde{\Delta}}$ is an optimal solution of program (6) .

Proof of Theorem 5. For each $g \in \mathcal{G}$, the scalar $\tilde{\varphi}^{g} \square$ is equal to the optimal value of program (7) with the incorporated upper bound $L$ on the objective function. If $g \in \mathcal{G}^{0}$, the bound $L$ is redundant and we have $\tilde{\varphi}^{g}=\operatorname{Eff}^{g}\left(X_{o}^{q}, Y_{o}^{q}\right)=\operatorname{Eff}^{M}\left(X_{o}^{q}, Y_{o}^{q}\right)$. If $g \in \mathcal{G} \backslash \mathcal{G}^{0}$, we have $\operatorname{Eff}^{M}\left(X_{o}^{q}, Y_{o}^{q}\right)<\tilde{\varphi}^{g} \leq L$.

Proof of Theorem 6. In line with Podinovski and Førsund (2010), define the output response function

$$
\psi(a)=\max \left\{b \mid\left(a X_{o}, b Y_{o}\right) \in T^{M}\right\}=\max _{g \in \mathcal{G}^{0}}\left(\max \left\{b \mid\left(a X_{o}, b Y_{o}\right) \in T^{g}\right\}\right) \triangleq \max _{g \in \mathcal{G}^{0}} \psi^{g}(a) .
$$

As follows from formula (3) in Podinovski and Førsund (2010), $\varepsilon_{o}^{g+}=\left(\psi^{g}\right)_{+}^{\prime}(1)$, for all $g \in \mathcal{G}^{0}$. Then $\varepsilon_{o}^{M+}=\max _{g \in \mathcal{G}^{0}}\left(\psi^{g}\right)^{\prime}{ }_{+}(1)=\max _{g \in \mathcal{G}^{0}} \varepsilon_{o}^{g+}$. The case of $\varepsilon_{o}^{M-}$ is similar.

Proof of Theorem 7. The proof follows by repeating Lemmas 2 and 3 proved for programs (4) and (5). In these lemmas and their proofs, we only need to change notation $\theta$ to $\alpha$, and programs (4) and (5) to programs (15) and (16), respectively. 


\section{References}

Afsharian, M., Ahn, H. (2014). The Luenberger indicator and directions of measurement: A bottomsup approach with an empirical illustration to German savings banks. International Journal of Production Research, 52(20), 6216-6233.

Afsharian, M., Ahn, H., Alirezaee, M. (2015). Developing selective proportionality on the FDH models: New insight on the proportionality axiom. International Journal of Information and Decision Sciences, 7(2), 99-114.

Agrell, P. J., Tind, J. (2001). A dual approach to nonconvex frontier models. Journal of Productivity Analysis, 16(2), 129-147.

Asmild, M. (2015). Frontier differences and the global Malmquist index. In J. Zhu (Ed.), Data envelopment analysis: A handbook of models and methods (pp. 447-461). New York: Springer Science + Business Media.

Banker, R. D., Charnes A., Cooper W. W. (1984). Some models for estimating technical and scale inefficiencies in data envelopment analysis. Management Science 30(9), 1078-1092.

Banker, R. D., Thrall, R. M. (1992). Estimation of returns to scale using data envelopment analysis. European Journal of Operational Research 62(1), 74-84.

Battese, G. E., Rao, D. P., O'Donnell, C. J. (2004). A metafrontier production function for estimation of technical efficiencies and technology gaps for firms operating under different technologies. Journal of Productivity Analysis, 21(1), 91-103.

Chambers, R. G., Chung, Y., Färe, R. (1998). Profit, directional distance functions, and Nerlovian efficiency. Journal of Optimization Theory and Applications, 98(2), 351-364.

Charnes, A., Cooper, W. W., Rhodes, E. (1978). Measuring the efficiency of decision making units. European Journal of Operational Research 2(6), 429-444.

Cooper, W. W., Seiford. L. M., Tone, K. (2007). Data envelopment analysis: A comprehensive text with models, applications, references and DEA-solver software. $\left(2^{\text {nd }}\right.$ ed.). New York: Springer Science + Business Media.

De Witte, K., Marques, R. C. (2009). Capturing the environment, a metafrontier approach to the drinking water sector. International Transactions in Operational Research, 16(2), 257-271.

Deprins, D., Simar, L., Tulkens, H. (1984). Measuring labor-efficiency in post offices. In M. Marchand, P. Pestieau, H. Tulkens (Eds.), The performance of public enterprises: Concepts and measurements (pp. 243-267). Amsterdam: North-Holland.

Dyson, R. G., Allen, R., Camanho, A. S., Podinovski, V. V., Sarrico, C. S., Shale, E. A. (2001). Pitfalls and protocols in DEA. European Journal of Operational Research, 132(2), 245-259.

Huang, C. W., Ting, C. T., Lin, C. H., Lin, C. T. (2013). Measuring non-convex metafrontier efficiency in international tourist hotels. Journal of the Operational Research Society, 64(2), 250259. 
Førsund, F. R., Hjalmarsson, L. (2004). Calculating scale elasticity in DEA models. Journal of the Operational Research Society, 55(10), 1023-1038.

Kerstens, K., O’Donnell, C. J., Van de Woestyne, I. (2015) Frontier metatechnologies and convexity: A restatement. Discussion paper LEM 2015-08. IESEG School of Management, France.

Kontolaimou, A., Tsekouras, K. (2010). Are cooperatives the weakest link in European banking? A non-parametric metafrontier approach. Journal of Banking \& Finance, 34(8), 1946-1957.

Kuosmanen, T. (2005). Weak disposability in nonparametric productivity analysis with undesirable outputs. American Journal of Agricultural Economics, 87(4), 1077-1082.

Kuosmanen, T., Podinovski, V. V. (2009). Weak disposability in nonparametric production analysis: Reply to Färe and Grosskopf. American Journal of Agricultural Economics, 91(2), 539-545.

Leleu, H. (2006). A linear programming framework for free disposal hull technologies and cost functions: Primal and dual models. European Journal of Operational Research, 168(2), 340-344.

O’Donnell, C. J., Rao, D. P., Battese, G. E. (2008). Metafrontier frameworks for the study of firmlevel efficiencies and technology ratios. Empirical Economics, 34(2), 231-255.

Podinovski, V. V. (2004a). Bridging the gap between the constant and variable returns-to-scale models: Selective proportionality in data envelopment analysis. Journal of the Operational Research Society, 55(3), 265-276.

Podinovski, V. V. (2004b). Production trade-offs and weight restrictions in data envelopment analysis. Journal of the Operational Research Society, 55(12), 1311-1322.

Podinovski, V.V. (2017) Returns to scale in convex production technologies. European Journal of Operational Research, 258(3), 970-982.

Podinovski, V.V., Chambers, R.G., Atici, K. B., Deineko, I. D. (2016). Marginal values and returns to scale for nonparametric production frontiers. Operations Research, 64(1), 236-250.

Podinovski, V.V., Førsund, F.R. (2010) Differential characteristics of efficient frontiers in data envelopment analysis. Operations Research, 58(6), 1743-1754.

Portela, M. C. A. S., Thanassoulis, E., Horncastle, A., Maugg, T. (2011). Productivity change in the water industry in England and Wales: Application of the meta-Malmquist index. Journal of the Operational Research Society, 62(12), 2173-2188.

Tiedemann, T., Francksen, T., Latacz-Lohmann, U. (2011). Assessing the performance of German Bundesliga football players: A non-parametric metafrontier approach. Central European Journal of Operations Research, 19(4), 571-587.

Zhang, N., Zhou, P., Choi, Y. (2013). Energy efficiency, CO2 emission performance and technology gaps in fossil fuel electricity generation in Korea: A meta-frontier non-radial directional distance function analysis. Energy Policy, 56, 653-662.

Zhang, N., Wei, X. (2015). Dynamic total factor carbon emissions performance changes in the Chinese transportation industry. Applied Energy, 146, 409-420. 